\title{
ESTIMATES OF HEAT KERNELS FOR NON-LOCAL REGULAR DIRICHLET FORMS
}

\author{
ALEXANDER GRIGOR'YAN, JIAXIN HU, AND KA-SING LAU
}

\begin{abstract}
In this paper we present new heat kernel upper bounds for a certain class of non-local regular Dirichlet forms on metric measure spaces, including fractal spaces. We use a new purely analytic method where one of the main tools is the parabolic maximum principle. We deduce an off-diagonal upper bound of the heat kernel from the on-diagonal one under the volume regularity hypothesis, restriction of the jump kernel and the survival hypothesis. As an application, we obtain two-sided estimates of heat kernels for non-local regular Dirichlet forms with finite effective resistance, including settings with the walk dimension greater than 2 .
\end{abstract}

\section{Contents}

1. Introduction

2. Terminology and main results

3. Tail estimates for quasi-local Dirichlet forms

4. Heat semigroup of the truncated Dirichlet form

5. Proof of Theorem 2.1

6. Heat kernel bounds using effective resistance

7. Appendix A: Parabolic maximum principle

8. Appendix B: List of lettered conditions

\section{INTRODUCTION}

We are concerned with heat kernel estimates for a class of non-local regular Dirichlet forms. Let $(M, d)$ be a locally compact separable metric space and let $\mu$ be a Radon measure on $M$ with full support. The triple $(M, d, \mu)$ will be referred to as a metric measure space. Let $(\mathcal{E}, \mathcal{F})$ be a regular Dirichlet form in $L^{2}(M, \mu)$. As is well known (cf. [11]), any regular Dirichlet form has the generator $\mathcal{L}$ that is a non-positive definite self-adjoint operator in $L^{2}(M, \mu)$, which in turn gives rise to

Received by the editors September 23, 2011 and, in revised form, November 13, 2012.

2010 Mathematics Subject Classification. Primary 47D07; Secondary 28A80, 60J35.

Key words and phrases. Heat kernel, non-local Dirichlet form, effective resistance.

The first author was supported by SFB 701 of the German Research Council (DFG) and the Grants from the Department of Mathematics and IMS of CUHK.

The second author was supported by NSFC (Grant No. 11071138, 11271122), SFB 701 and the HKRGC Grant of CUHK. The second author is the corresponding author.

The third author was supported by the HKRGC Grant, the Focus Investment Scheme of CUHK, and also by the NSFC (no. 11171100, 11371382). 
the heat semigroup $P_{t}=e^{t \mathcal{L}}$ and an associated Hunt process $\left(\left\{X_{t}\right\}_{t \geq 0},\left\{\mathbb{P}_{x}\right\}_{x \in M}\right)$ on $M$.

If the operator $P_{t}$ is an integral operator, that is, has the integral kernel $p_{t}(x, y)$, then the latter is called the heat kernel. At the same time, $p_{t}(x, y)$ is the transition density of the process $\left\{X_{t}\right\}$. The question of the existence and the estimates of the heat kernel has attracted considerable attention in the literature. The estimates of the heat kernel could then be used for many applications, in particular, for obtaining information about the path properties of $\left\{X_{t}\right\}$ and the spectral properties of the operator $\mathcal{L}$.

Depending on the setting one expects different types of estimates of the heat kernel $p_{t}(x, y)$. For example, in $\mathbb{R}^{n}$ with the classical Dirichlet form

$$
\mathcal{E}(u, u)=\int_{\mathbb{R}^{n}}|\nabla u|^{2} d x
$$

whose generator is the classical Laplace operator $\Delta$, the heat kernel is the GaussWeierstrass function

$$
p_{t}(x, y)=\frac{1}{(4 \pi t)^{n / 2}} \exp \left(-\frac{|x-y|^{2}}{4 t}\right)
$$

that is also the transition density of Brownian motion. For the Dirichlet form

$$
\mathcal{E}(u, u)=\int_{\mathbb{R}^{n}} a_{i j}(x) \frac{\partial u}{\partial x_{i}} \frac{\partial u}{\partial x_{j}} d x
$$

where $\left(a_{i j}(x)\right)$ is a symmetric uniformly elliptic matrix, the generator is the operator

$$
\mathcal{L}=\frac{\partial}{\partial x_{i}}\left(a_{i j} \frac{\partial}{\partial x_{j}}\right)
$$

and the heat kernel admits (cf. [1]) the two-sided Gaussian bounds

$$
p_{t}(x, y) \asymp \frac{C}{t^{n / 2}} \exp \left(-\frac{|x-y|^{2}}{c t}\right) .
$$

Similar bounds hold on some classes of Riemannian manifolds (see [14, 33]). Note that in the above examples the Dirichlet form is local and, hence, the corresponding Hunt process is a diffusion.

Local Dirichlet forms may also be defined on some singular spaces like fractals, where the definition is implicit and uses the self-similarity structure (cf. [2], 3], [21], 27, 32]). On some classes of fractals the heat kernel is known to exist and to satisfy the following sub-Gaussian estimates:

$$
p_{t}(x, y) \asymp \frac{C}{t^{\alpha / \beta}} \exp \left(-\left(\frac{d(x, y)}{c t^{1 / \beta}}\right)^{\beta /(\beta-1)}\right),
$$

where $\alpha>0$ and $\beta>1$ are some parameters that characterize the underlying space in question, and $d(x, y)$ is an appropriate distance function.

Consider the following example of a non-local Dirichlet form in $\mathbb{R}^{n}$ :

$$
\mathcal{E}(u, u)=\int_{\mathbb{R}^{n}} \int_{\mathbb{R}^{n}} \frac{(u(x)-u(y))^{2}}{|x-y|^{n+\beta}} d x d y,
$$


where $0<\beta<2$. The generator of this form is const $(-\Delta)^{\beta / 2}$, and the heat kernel admits the bounds

$$
p_{t}(x, y) \asymp \frac{1}{t^{n / \beta}}\left(1+\frac{|x-y|}{t^{1 / \beta}}\right)^{-(n+\beta)} .
$$

The associated Hunt process is the symmetric stable process of index $\beta$, that is, a jump process.

Similarly, if $\mathcal{L}$ denotes the generator of diffusion with the heat kernel (1.1), then the operator $-(-\mathcal{L})^{\gamma}$ for any $0<\gamma<1$ is the generator of a non-local Dirichlet form, and its heat kernel admits the estimate

$$
p_{t}(x, y) \asymp \frac{1}{t^{\alpha / \beta^{\prime}}}\left(1+\frac{d(x, y)}{t^{1 / \beta^{\prime}}}\right)^{-\left(\alpha+\beta^{\prime}\right)},
$$

where $\beta^{\prime}=\beta \gamma$ (see, for example, [13], 26], 36]). It was shown in 20] that (1.1) and (1.2) exhaust all possible two-sided estimates of heat kernels of self-similar type.

The purpose of this paper is to give equivalent conditions for upper bounds of the heat kernels of non-local type.

Let us return to the general setup of a metric measure space $(M, d, \mu)$ equipped with a regular Dirichlet form $(\mathcal{E}, \mathcal{F})$. Assume in addition that $(\mathcal{E}, \mathcal{F})$ is conservative, that is, $P_{t} 1 \equiv 1$. Denote by $V(x, r)$ the measure of the metric ball $B(x, r)$. We assume throughout that all metric balls are precompact. In particular, this implies that $V(x, r)$ is finite.

Let $\alpha, \beta$ be fixed positive numbers, and let $C$ denote a positive constant that can be different at different occurrences. Let us state the following conditions that in general may be true or not.

$\left(\mathbf{V}_{\leq}\right)$: Upper $\alpha$-regularity: For all $x \in M$ and all $r>0$,

$$
V(x, r) \leq C r^{\alpha} \text {. }
$$

(DUE) : On-diagonal upper estimate: The heat kernel $p_{t}$ exists and satisfies the on-diagonal upper estimate

$$
p_{t}(x, y) \leq \frac{C}{t^{\alpha / \beta}},
$$

for all $t>0$ and $\mu$-almost all $x, y \in M$.

(UE) : Upper estimate of non-local type: The heat kernel $p_{t}$ exists and satisfies the off-diagonal upper estimate

$$
p_{t}(x, y) \leq \frac{C}{t^{\alpha / \beta}}\left(1+\frac{d(x, y)}{t^{1 / \beta}}\right)^{-(\alpha+\beta)}
$$

for all $t>0$ and $\mu$-almost all $x, y \in M$.

$\left(\mathbf{U E}_{l o c}\right)$ : Upper estimate of local type: The heat kernel $p_{t}$ exists and satisfies the off-diagonal upper estimate

$$
p_{t}(x, y) \leq \frac{C}{t^{\alpha / \beta}} \exp \left(-\left(\frac{d(x, y)}{c t^{1 / \beta}}\right)^{\beta /(\beta-1)}\right)
$$

for all $t>0$ and $\mu$-almost all $x, y \in M$, where $\beta>1$.

(S) : Survival estimate: There exist constants $\varepsilon, \delta \in(0,1)$ such that, for all $x \in M$, all balls $B(x, r)$ and all $t^{1 / \beta} \leq \delta r$,

$$
\mathbb{P}_{x}\left(\tau_{B(x, r)} \leq t\right) \leq \varepsilon,
$$

where $\tau_{\Omega}$ is the first exit time of the process $\left\{X_{t}\right\}$ from a set $\Omega$. 
Note that $\mathbb{P}_{x}\left(\tau_{B(x, r)} \leq t\right)$ is the probability of $\left\{X_{t}\right\}$ to leave $B(x, r)$ before time $t$. The smallness of this probability as is stated in $(S)$ means a high probability of the process staying in $B(x, r)$ up to time $t$. The latter can be considered as the probability of survival of the process up to the time $t$ assuming that outside $B(x, r)$ the process gets killed.

The above definition of condition $(S)$ is a bit loose because in general $\mathbb{P}_{x}$ may not be defined for all $x \in M$. A rigorous analytic definition of $(S)$ in terms of the heat semigroup will be given in Section 2

Condition $\left(V_{\leq}\right)$determines the value of the parameter $\alpha$, while condition $(S)$ determines $\beta$ that is a space/time scaling parameter. In the fractal literature $\beta$ is called the walk dimension.

Under the standing assumption $\left(V_{\leq}\right)$, the following characterization of $\left(U E_{l o c}\right)$ is known as

$$
\left(U E_{l o c}\right) \Leftrightarrow(D U E)+(S)+(\text { "locality"), }
$$

where "locality" means that the form $(\mathcal{E}, \mathcal{F})$ is local (see [16] and Remark 2.5 below). In the present paper we prove a similar characterization of the non-local upper estimate $(U E)$ by relaxing the condition of the locality of $(\mathcal{E}, \mathcal{F})$. Recall that by a theorem of Beurling and Deny, any regular conservative Dirichlet form admits a decomposition

$$
\mathcal{E}(u, v)=\mathcal{E}^{(L)}(u, v)+\mathcal{E}^{(J)}(u, v),
$$

where $\mathcal{E}^{(L)}$ is a local part and

$$
\mathcal{E}^{(J)}(u, v)=\int_{M \times M \backslash \operatorname{diag}}(u(x)-u(y))(v(x)-v(y)) d j(x, y)
$$

is a jump part with a jump measure $j$ defined on $M \times M \backslash$ diag. In our setting the jump measure $j$ will have a density with respect to $\mu \times \mu$, which will be denoted by $J(x, y)$, and so the jump part $\mathcal{E}^{(J)}$ becomes

$$
\mathcal{E}^{(J)}(u, v)=\iint_{M \times M}(u(x)-u(y))(v(x)-v(y)) J(x, y) d \mu(y) d \mu(x) .
$$

Let us consider the following condition:

$\left(\mathbf{J}_{\leq}\right)$: The jump density exists and admits the estimate

$$
J(x, y) \leq C d(x, y)^{-(\alpha+\beta)},
$$

for $\mu$-almost all $x, y \in M$.

In the case of a local form $(\mathcal{E}, \mathcal{F})$, we have $J=0$ so that $\left(J_{\leq}\right)$is trivially satisfied. In general, $\left(J_{\leq}\right)$restricts the long jumps and can be regarded as a measure of nonlocality.

Our main result (Theorem 2.3 below) states that, under the standing assumption $\left(V_{\leq}\right)$, the following equivalence holds:

$$
(U E) \Leftrightarrow(D U E)+(S)+\left(J_{\leq}\right) .
$$

We would like to emphasize that it is an analogy with (1.3) where the locality was used instead of $\left(J_{\leq}\right)$. 
Let us comment on the individual conditions in (1.7). It is well known that $(D U E)$ is equivalent to a certain Nash functional inequality for the Dirichlet form $(\mathcal{E}, \mathcal{F})$ (cf. [8]). On the other hand, the Nash inequality is known to be true provided

$$
J(x, y) \geq c d(x, y)^{-(\alpha+\beta)}
$$

and $V(x, r) \geq c r^{\alpha}$ (see 24 and Proposition 2.6 below). Hence, under these conditions one has also $(D U E)$. It seems that the upper bound $\left(J_{\leq}\right)$of the jump density alone does not imply $(D U E)$.

An equivalence somewhat similar to (1.7) was proved in [6], where instead of condition $(S)$ one used a stronger condition

$$
\mathbb{P}_{x}\left(\tau_{B(x, r)} \leq t\right) \leq C \frac{t}{r^{\beta}}
$$

for all $t, r>0$, and instead of $\left(J_{\leq}\right)$a certain property of the sample paths that also restricts the long jumps, but in different terms.

Under the assumption $\beta<2$ and some additional hypotheses, another result of [6] states that, for the pure jump Dirichlet form (1.5),

$$
(U E) \Leftrightarrow(D U E)+\left(J_{\leq}\right) .
$$

Hence, in this case the hypothesis $(S)$ in (1.7) can be dropped. Moreover, if both condition $(V)$ (that is, $V(x, r) \asymp r^{\alpha}$ ) and condition $(J)$ (that is, $J(x, y) \asymp$ $\left.d(x, y)^{-(\alpha+\beta)}\right)$ hold, then $(U E)$ is true without any further hypothesis; see [9, 10,

In general if $\beta \geq 2$ (as may happen on fractal spaces), it is not known whether $(S)$ can be dropped.

However, conditions $(S)$ and $(D U E)$ in (1.7) can be verified under certain hypotheses about effective resistance $R(x, y)$ (cf. the definition at the beginning of Section [6 as well as [27, 28, [29]). Assume that $0<\alpha<\beta$, and consider the following condition, named $(R)$ :

$$
R(x, y) \asymp d(x, y)^{\beta-\alpha} .
$$

This condition $(R)$ implies that the corresponding process is recurrent; see (6.47) below.

If $(\mathcal{E}, \mathcal{F})$ is parabolic (cf. Definition 6.3) and condition $(V)$ is satisfied, we prove in Theorem 6.17 that

$$
(U E)+(N L E) \Leftrightarrow(R)+\left(J_{\leq}\right),
$$

where $(N L E)$ stands for the near diagonal lower estimate of the heat kernel (cf. Section 6.7). Let us mention for comparison that, under the same standing assumptions,

$$
\left(U E_{l o c}\right)+(N L E) \Leftrightarrow(R)+(\text { "locality") }
$$

(cf. [31, Theorem 3.1] and Corollary 6.18).

The techniques for obtaining heat kernel bounds for non-local Dirichlet forms have been developed by a number of authors; see for example [4, 6, 7, 9, 10] and the references therein. The basic approach to obtaining heat kernel upper estimates used in these papers consists of two steps. The first step is to obtain the heat kernel upper bounds for a truncated Dirichlet form, that is, in the case when the jump density $J(x, y)$ has a bounded range. In this case one uses the Davies method as it was presented in the seminal work [8] and where the cut-off functions of form $\left(\lambda-d\left(x_{0}, x\right)\right)_{+}$were used (where $\lambda$ is a positive constant). This method can be used as long as the cut-off functions belong to the domain of the Dirichlet form, 
which is the case only when $\beta<2$ (hence, if $\beta \geq 2$, then this method does not work).

The second step is to obtain heat kernel estimates for the original Dirichlet form by comparing the heat semigroup of the truncated Dirichlet form with the original heat semigroup. We remark that while the first step was done by purely analytic means, the second step in the above-mentioned papers used a probabilistic argument.

In this article, we develop an alternative approach to obtaining upper bounds that is new in the following two aspects:

(1) We give a new method of obtaining heat kernel estimates for truncated Dirichlet forms without restriction on the walk dimension.

(2) We prove new simple relations between the truncated and original heat semigroups, using the parabolic maximum principle developed in [15]. This argument is purely analytic.

The structure of the paper is as follows. In Section 2 we state the main results of this paper: Theorems 2.1 and 2.3. In Section 3 we obtain tail estimates for non-local Dirichlet forms, the main technical results here being Theorem 3.1 and Corollary 3.2, In Section 4, we obtain heat kernel estimates for the truncated heat semigroup; see Theorem 4.9. In Section 5, we prove the main theorem, Theorem 2.1 ,

In Section 6 we apply Theorem 2.1 to obtain two-sided heat kernel bounds using effective resistance, where the main results are Theorems 6.13 and 6.17 .

In Appendix $\mathrm{A}$ we state for convenience of the reader the parabolic maximum principle that is the main technical tool used in this paper. In Appendix B we list all lettered hypotheses used.

Notation. The letters $c, c_{1}, C_{1}$, etc. denote positive constants whose values are unimportant and may differ at different occurrences. The relation $f \asymp g$ between two non-negative functions $f, g$ means that there is a constant $C \geq 1$ such that $C^{-1} f \leq g \leq C f$ for a specified range of the variables.

\section{TERMinOLOGY AND MAIN RESUltS}

Let $(M, d, \mu)$ be a metric measure space that is unbounded. Recall that a Dirichlet form $(\mathcal{E}, \mathcal{F})$ in $L^{2}(M, \mu)$ is a symmetric, non-negative definite, bilinear form $\mathcal{E}: \mathcal{F} \times \mathcal{F} \rightarrow \mathbb{R}$ defined on a dense subspace $\mathcal{F}$ of $L^{2}(M, \mu)$, which satisfies in addition the following properties:

(1) Closedness: $\mathcal{F}$ is a Hilbert space with respect to the following inner product:

$$
\mathcal{E}_{1}(f, g):=\mathcal{E}(f, g)+(f, g) .
$$

(2) The Markov property: if $f \in \mathcal{F}$, then also $\tilde{f}:=(f \wedge 1)_{+}$belongs to $\mathcal{F}$ and $\mathcal{E}(\widetilde{f}) \leq \mathcal{E}(f)$, where $\mathcal{E}(f):=\mathcal{E}(f, f)$.

Then $(\mathcal{E}, \mathcal{F})$ has the generator $\mathcal{L}$ that is a non-positive definite, self-adjoint operator on $L^{2}(M, \mu)$ with domain $\mathcal{D} \subset \mathcal{F}$ such that

$$
\mathcal{E}(f, g)=(-\mathcal{L} f, g)
$$

for all $f \in \mathcal{D}$ and $g \in \mathcal{F}$. The generator $\mathcal{L}$ determines the heat semigroup $\left\{P_{t}\right\}_{t \geq 0}$ by $P_{t}=e^{t \mathcal{L}}$ in the sense of functional calculus of self-adjoint operators. It is known 
that $\left\{P_{t}\right\}_{t>0}$ is a strongly continuous, contractive, symmetric semigroup in $L^{2}$, and is Markovian, that is, $0 \leq P_{t} f \leq 1$ for any $t>0$ if $0 \leq f \leq 1$.

The Markovian property of the heat semigroup implies that the operator $P_{t}$ preserves the inequalities between functions, which allows us to use monotone limits to extend $P_{t}$ from $L^{2}$ to $L^{\infty}$ (in fact, $P_{t}$ extends to any $L^{q}, 1 \leq q \leq \infty$, as a contraction; cf. [11, p. 37]). In particular, $P_{t} 1$ is defined. The form $(\mathcal{E}, \mathcal{F})$ is called conservative if $P_{t} 1=1$ for every $t>0$.

The Dirichlet form $(\mathcal{E}, \mathcal{F})$ can be recovered from the heat semigroup as follows. For any $f \in L^{2}$, the function

$$
t \mapsto \frac{1}{t}\left(f-P_{t} f, f\right)
$$

is increasing as $t$ is decreasing. In particular, it has the limit as $t \rightarrow 0$. It turns out that the limit is finite if and only if $f \in \mathcal{F}$, and

$$
\lim _{t \rightarrow 0+} \frac{1}{t}\left(f-P_{t} f, f\right)=\mathcal{E}(f) .
$$

Fix some $\rho \in[0,+\infty)$. A Dirichlet form $(\mathcal{E}, \mathcal{F})$ is said to be $\rho$-local if $\mathcal{E}(f, g)=0$ for any two functions $f, g \in \mathcal{F}$ with compact supports such that

$$
\operatorname{dist}(\operatorname{supp} f, \operatorname{supp} g)>\rho \text {. }
$$

We call a form $(\mathcal{E}, \mathcal{F})$ quasi-local if it is $\rho$-local for some $0<\rho<\infty$.

For a non-empty open $\Omega \subset M$, let $\mathcal{F}(\Omega)$ be the closure of $\mathcal{F} \cap C_{0}(\Omega)$ in the norm of $\mathcal{F}$. It is known that if $(\mathcal{E}, \mathcal{F})$ is regular, then $(\mathcal{E}, \mathcal{F}(\Omega))$ is a regular Dirichlet form in $L^{2}(\Omega, \mu)$ since $\mathcal{F}(\Omega)$ is dense in $L^{2}(\Omega, \mu)$. Denote by $P_{t}^{\Omega}$ the heat semigroup of $(\mathcal{E}, \mathcal{F}(\Omega))$.

Let us give a probabilistic interpretation of the heat semigroups $P_{t}$ and $P_{t}^{\Omega}$. For any regular Dirichlet form $(\mathcal{E}, \mathcal{F})$, there is an associated Hunt process. Denote by $X_{t}, t \geq 0$, the trajectories of a process and by $\mathbb{P}_{x}, x \in M$, the probability measure in the space of trajectories emanating from the point $x$. Denote by $\mathbb{E}_{x}$ the expectation of the probability measure $\mathbb{P}_{x}$. Then the relation between the Dirichlet form and the associated Hunt process is given by the following identity:

$$
P_{t} f(x)=\mathbb{E}_{x}\left[f\left(X_{t}\right)\right]
$$

for all bounded (or non-negative) Borel functions $f$ and all $t>0$, and for $\mu$ almost all $x \in M$ (note that $P_{t} f$ is a function from $L^{\infty}$ and, hence, is defined up to a set of measure zero, whereas $\mathbb{E}_{x} f\left(X_{t}\right)$ is defined pointwise for all $\left.x \in M\right)$. By [11, Theorem 7.2.1, p. 380], such a process always exists but, in general, is not unique. For a non-empty subset $\Omega$ of $M$, one has the following identity (see [11, p. 153, eq. (4.1.2)]): for all bounded (or non-negative) Borel functions $f$ and for all $t>0$,

$$
P_{t}^{\Omega} f(x)=\mathbb{E}_{x}\left[\mathbf{1}_{\left\{t<\tau_{\Omega}\right\}} f\left(X_{t}\right)\right],
$$

where $\tau_{\Omega}$ is the first exit time $\tau_{\Omega}$ defined by

$$
\tau_{\Omega}=\inf \left\{t>0: X_{t} \notin \Omega\right\} .
$$

In particular, if $f=\mathbf{1}_{\Omega}$, we see from (2.3) that

$$
P_{t}^{\Omega} \mathbf{1}_{\Omega}(x)=\mathbb{P}_{x}\left(t<\tau_{\Omega}\right) .
$$


A family $\left\{p_{t}\right\}_{t>0}$ of $\mu \times \mu$-measurable functions on $M \times M$ is called the heat kernel of the form $(\mathcal{E}, \mathcal{F})$ if $p_{t}$ is the integral kernel of the operator $P_{t}$, that is, for any $t>0$ and for any $f \in L^{2}(M, \mu)$,

$$
P_{t} f(x)=\int_{M} p_{t}(x, y) f(y) d \mu(y)
$$

for $\mu$-almost all $x \in M$, where $\left\{P_{t}\right\}_{t \geq 0}$ is the heat semigroup of $(\mathcal{E}, \mathcal{F})$ as mentioned above.

For any $x \in M$ and $r>0$, denote by

$$
B(x, r)=\{y \in M: d(y, x)<r\}
$$

a metric ball in $M$ with center $x$ and radius $r$. We assume throughout that all balls are precompact. Set $V(x, r):=\mu(B(x, r))$.

In addition to conditions $(D U E)$ and $(U E)$ defined in the Introduction, consider the following condition:

$(\mathbf{U E} \Phi)$ : The heat kernel $p_{t}$ exists and there exist $C, \alpha>0, \beta>0$ such that

$$
p_{t}(x, y) \leq \frac{C}{t^{\alpha / \beta}} \Phi\left(\frac{d(x, y)}{t^{1 / \beta}}\right)
$$

for all $t>0$ and $\mu$-almost all $x, y \in M$, where $\Phi:[0, \infty) \rightarrow[0, \infty)$ is a continuous, non-increasing function such that

$$
\int_{0}^{\infty} s^{\alpha-1} \Phi(s) d s<\infty .
$$

Let us restate in analytic terms the survival condition mentioned in the Introduction:

(S) : Survival estimate. There exist constants $\varepsilon, \delta \in(0,1)$ and $\beta>0$ such that, for all balls $B=B\left(x_{0}, r\right)$ and for all $t^{1 / \beta} \leq \delta r$,

$$
1-P_{t}^{B} \mathbf{1}_{B}(x) \leq \varepsilon \text { for } \mu \text {-almost all } x \in \frac{1}{4} B,
$$

where $\lambda B=B\left(x_{0}, \lambda r\right)$.

By (2.5), we have that

$$
\begin{aligned}
1-P_{t}^{B} \mathbf{1}_{B}(x) & =1-\mathbb{P}_{x}\left(t<\tau_{B}\right) \\
& =\mathbb{P}_{x}\left(\tau_{B} \leq t\right),
\end{aligned}
$$

which implies the equivalence of the two definitions of $(S)$.

Finally, consider two more conditions.

(T) : Tail estimate. There exist constants $\varepsilon, \delta \in(0,1)$ and $\beta>0$ such that, for all balls $B=B\left(x_{0}, r\right)$ and for all $t^{1 / \beta} \leq \delta r$,

$$
P_{t} \mathbf{1}_{B^{c}}(x) \leq \varepsilon \text { for } \mu \text {-almost all } x \in \frac{1}{4} B \text {. }
$$

$\left(\mathbf{T}_{\text {strong }}\right)$ : Strong tail estimate. There exist constants $c>0$ and $\beta>0$ such that, for all balls $B=B\left(x_{0}, r\right)$ and for all $t>0$,

$$
P_{t} \mathbf{1}_{B^{c}}(x) \leq \frac{c t}{r^{\beta}} \text { for } \mu \text {-almost all } x \in \frac{1}{4} B .
$$

Clearly, we have that $\left(T_{\text {strong }}\right) \Rightarrow(T)$.

We now state the main technical result of this paper. 
Theorem 2.1. Let $(M, d, \mu)$ be a metric measure space with precompact balls, and let $(\mathcal{E}, \mathcal{F})$ be a regular conservative Dirichlet form in $L^{2}(M, \mu)$ with jump density $J$. Then the following implication holds:

$$
\left(V_{\leq}\right)+(D U E)+\left(J_{\leq}\right)+(S) \Rightarrow(U E) .
$$

The proof of Theorem 2.1 will be given in Section 5 .

Remark 2.2. Also define the condition

$(\mathbf{V})$ : There exist constants $c, C, \alpha>0$ such that, for all $x \in M$ and all $r>0$,

$$
c r^{\alpha} \leq V(x, r) \leq C r^{\alpha} \text {. }
$$

If the measure $\mu$ satisfies $(V)$, then $\mu$ is called $\alpha$-regular.

By [17, Theorem 3.2], if $(\mathcal{E}, \mathcal{F})$ is conservative, then

$$
\left(V_{\leq}\right)+(U E) \Rightarrow(V) .
$$

Hence, the hypotheses of Theorem 2.1 imply that $\mu$ is $\alpha$-regular.

The following main theorem provides convenient equivalent conditions for $(U E)$ and is a combination of Theorem 2.1 with previously known results.

Theorem 2.3. Let $(M, d, \mu)$ be a metric measure space with precompact balls, and let $(\mathcal{E}, \mathcal{F})$ be a regular conservative Dirichlet form in $L^{2}(M, \mu)$ with jump density $J$. If $\left(V_{\leq}\right)$holds, then the following equivalences are true:

$$
\begin{aligned}
(U E) & \Leftrightarrow(U E \Phi)+\left(J_{\leq}\right) \\
& \Leftrightarrow(D U E)+\left(J_{\leq}\right)+(T) \\
& \Leftrightarrow(D U E)+\left(J_{\leq}\right)+(S) \\
& \Leftrightarrow(D U E)+\left(J_{\leq}\right)+\left(T_{\text {strong }}\right) .
\end{aligned}
$$

Proof. Observe that the implication $(U E) \Rightarrow\left(J_{\leq}\right)$holds by [6, p. 150], and $(U E) \Rightarrow$ $(U E \Phi)$ is trivial by taking $\Phi(s)=(1+s)^{-(\alpha+\beta)}$. The implication $(U E \Phi) \Rightarrow$ $(D U E)$ is obvious. The implication $(U E \Phi) \Rightarrow(T)$ was proved in 17, formula (3.6), p. 2072]. Since $(\mathcal{E}, \mathcal{F})$ is conservative, the equivalence $(T) \Leftrightarrow(S)$ holds by [15. Theorem 3.1, p. 96]. By Theorem 2.1 we have

$$
(D U E)+\left(J_{\leq}\right)+(S) \Rightarrow(U E),
$$

which closes the cycle of implications, thus proving the first three equivalences.

Finally, the implication $(U E) \Rightarrow\left(T_{\text {strong }}\right)$ is true (see also [17, formula (3.6), p. 2072]), and hence

$$
\begin{aligned}
(U E) & \Rightarrow(D U E)+\left(J_{\leq}\right)+\left(T_{\text {strong }}\right) \\
& \Rightarrow(D U E)+\left(J_{\leq}\right)+(T) \Rightarrow(U E),
\end{aligned}
$$

which finishes the proof.

Remark 2.4. If $(\mathcal{E}, \mathcal{F})$ has only a jump part and $0<\beta<2$, then $\left(V_{\leq}\right)$and $\left(J_{\leq}\right)$ imply that $(\mathcal{E}, \mathcal{F})$ is conservative (see $[19,35]$ ).

Remark 2.5. Under the standing assumptions of Theorem 2.3 the following equivalence is also true:

$$
\left(U E_{l o c}\right) \Leftrightarrow(D U E)+(\text { "locality" })+(S) .
$$


Indeed, since $\left(U E_{l o c}\right)$ is stronger than $(U E)$, it implies $(D U E)$ and $(S)$ by Theorem 2.3. Next, $\left(U E_{l o c}\right) \Rightarrow$ ("locality") by [20, Lemma 3.1]. The opposite implication

$$
(D U E)+(\text { "locality" })+(S) \Rightarrow\left(U E_{l o c}\right)
$$

was proved in [16].

In order to state some consequence of Theorem 2.3, we need the following proposition.

Define first the following condition:

$\left(\mathbf{J}_{\geq}\right)$: There exist constants $C, \alpha, \beta>0$ such that, for $\mu$-almost all $x \neq y$,

$$
J(x, y) \geq C^{-1} d(x, y)^{-(\alpha+\beta)} .
$$

Proposition 2.6. Let $(M, d, \mu)$ be a metric measure space, and let $(\mathcal{E}, \mathcal{F})$ be a regular Dirichlet form in $L^{2}(M, \mu)$ with jump density $J$. Then

$$
(V)+\left(J_{\geq}\right) \Rightarrow(D U E) .
$$

Proof. As was proved in [24, Theorem 3.1], under $(V)$ the following inequality holds for all non-zero functions $u \in L^{1} \cap L^{2}$ :

$$
\int_{M} \int_{M} \frac{(u(x)-u(y))^{2}}{d(x, y)^{\alpha+\beta}} d \mu(x) d \mu(y) \geq c\|u\|_{2}^{2(1+\beta / \alpha)}\|u\|_{1}^{-2 \beta / \alpha},
$$

where $c$ is a positive constant. Using (1.4), (1.6) and $\left(J_{\geq}\right)$we obtain

$$
\begin{aligned}
\mathcal{E}(u) & =\mathcal{E}^{(L)}(u)+\mathcal{E}^{(J)}(u) \\
& \geq C \int_{M} \int_{M} \frac{(u(x)-u(y))^{2}}{d(x, y)^{\alpha+\beta}} d \mu(x) d \mu(y) \\
& \geq c\|u\|_{2}^{2(1+\beta / \alpha)}\|u\|_{1}^{-2 \beta / \alpha}
\end{aligned}
$$

for all $u \in \mathcal{F} \cap L^{1}$. Hence, $(D U E)$ follows by [8].

Corollary 2.7. Let $(M, d, \mu)$ be a metric measure space with precompact balls, and let $(\mathcal{E}, \mathcal{F})$ be a regular conservative Dirichlet form in $L^{2}(M, \mu)$ with jump density $J$. If $(V)$ holds and $J(x, y) \asymp d(x, y)^{-(\alpha+\beta)}$, then

$$
(U E) \Leftrightarrow(S) \text {. }
$$

Proof. Let us show that $(S) \Rightarrow(U E)$. Indeed, $(D U E)$ holds by Proposition 2.6 . Hence, $(U E)$ is satisfied by Theorem 2.3 . The opposite implication $(U E) \Rightarrow(S)$ also holds by Theorem 2.3 .

Therefore, if $(V)$ holds and $J(x, y) \asymp d(x, y)^{-(\alpha+\beta)}$, then in order to obtain off-diagonal upper bounds of heat kernels, one needs only to verify the survival condition $(S)$. We will show in Section 6 that the survival condition $(S)$ holds for a class of measure spaces with effective resistance metrics.

Remark 2.8. The upper estimate $(U E)$ is best possible for non-local forms in the following sense: if the heat kernel $p_{t}$ satisfies the estimate

$$
p_{t}(x, y) \leq \frac{1}{t^{\alpha / \beta}} \Phi\left(\frac{d(x, y)}{t^{1 / \beta}}\right)
$$


for all $t>0$ and $\mu$-almost all $x, y \in M$, where $\Phi$ is a continuous decreasing function on $[0,+\infty)$, then necessarily

$$
\Phi(s) \geq c(1+s)^{-(\alpha+\beta)}
$$

for some $c>0$ (see [20, Lemma 3.1]).

\section{TAIL ESTIMATES FOR QUASI-LOCAL DiRIChlet FORMS}

In this section we give the tail estimate for an arbitrary $\rho$-local regular Dirichlet form $\left(\mathcal{E}^{(\rho)}, \mathcal{F}^{(\rho)}\right)$ in $L^{2}(M, \mu)$. We use the superscript $(\rho)$ in the notation of this form in order to emphasize that the results of this section will be applied in Sections 4 and 5 for the Dirichlet form, also denoted by $\left(\mathcal{E}^{(\rho)}, \mathcal{F}^{(\rho)}\right)$, that is obtained by $\rho$ truncation of the jump density of the original Dirichlet form $(\mathcal{E}, \mathcal{F})$. However, in this section $\left(\mathcal{E}^{(\rho)}, \mathcal{F}^{(\rho)}\right)$ stands for any $\rho$-local regular Dirichlet form.

Denote by $\left\{Q_{t}^{\Omega}\right\}$ the heat semigroup of the form $\left(\mathcal{E}^{(\rho)}, \mathcal{F}^{(\rho)}(\Omega)\right)$ restricted to a non-empty open set $\Omega \subset M$. Denote by

$$
U_{\rho}=\{x: d(x, U)<\rho\}
$$

the $\rho$-neighborhood of a set $U \subset M$ if $\rho>0$, and let $U_{\rho}=U$ if $\rho=0$. We will use the following inequality obtained in [18, Corollary 4.8, Remark 4.10]. Let $\Omega, U$ be two open subsets of $M$ such that $U_{\rho}$ is precompact and $U_{\rho} \subset \Omega$. Then, for any $0 \leq f \in L^{\infty}(M)$ such that $\left.f\right|_{U} \equiv 0$ and for all $t>0$ and $\mu$-almost all $x \in U_{\rho}$,

$$
Q_{t}^{\Omega} f(x) \leq\left(1-Q_{t}^{U} \mathbf{1}_{U}(x)\right) \sup _{0<s \leq t}\left\|Q_{s}^{\Omega} f\right\|_{L^{\infty}\left(U_{\rho}\right)} .
$$

Theorem 3.1. Let $\phi(r, \cdot)$ be a non-decreasing function in $(0, \infty)$ for any $r \in(0, \infty)$. Assume that, for any ball $B=B(x, r)$ and for any $t \in\left(0, T_{0}\right)$ where $T_{0} \in(0, \infty]$,

$$
1-Q_{t}^{B} \mathbf{1}_{B} \leq \phi(r, t) \quad \text { in } \frac{1}{4} B .
$$

Then, for any ball $B=B(x, r), t \in\left(0, T_{0}\right)$ and any integer $k \geq 1$,

$$
Q_{t} \mathbf{1}_{B(x, k(r+\rho))^{c}} \leq \phi(r, t)^{k} \text { in } \frac{1}{4} B .
$$

Consequently, for any ball $B(x, R)$ with $R>\rho$ and $t \in\left(0, T_{0}\right)$, for any integer $k \geq 1$,

$$
Q_{t} \mathbf{1}_{B(x, k R)^{c}} \leq \phi(R-\rho, t)^{k-1} \text { in } B(x, R) .
$$

Proof. We will prove (3.3) by induction in $k$. Indeed, let $B_{k}=B(x, k(r+\rho))$. Then (3.3) holds for $k=1$, since by (3.2) we have in $\frac{1}{4} B$

$$
Q_{t} \mathbf{1}_{B_{1}^{c}} \leq 1-Q_{t} \mathbf{1}_{B_{1}} \leq 1-Q_{t}^{B} \mathbf{1}_{B} \leq \phi(r, t) .
$$

For the inductive step from $k$ to $k+1$, consider the function

$$
u(t, \cdot)=Q_{t} \mathbf{1}_{B_{k+1}^{c}} .
$$

Applying (3.1) with $\Omega=M, U=B$ and $f=\mathbf{1}_{B_{k+1}^{c}}$, we obtain the following inequality in $U_{\rho}=B_{1}$ :

$$
u(t, \cdot) \leq\left(1-Q_{t}^{B} \mathbf{1}_{B}\right) \sup _{0<s \leq t}\|u(s, \cdot)\|_{L^{\infty}\left(B_{1}\right)} .
$$

Note that, for every $z \in B_{1}$,

$$
B_{k+1}^{c} \subset B(z, k(r+\rho))^{c},
$$


and that, for $0<s \leq t$,

$$
\phi(r, s) \leq \phi(r, t) .
$$

Applying the inductive hypothesis for the ball $B(z, k(r+\rho))$, we obtain the following inequality in $B\left(z, \frac{1}{4} r\right)$ :

$$
u(s, \cdot)=Q_{s} \mathbf{1}_{B_{k+1}^{c}} \leq Q_{s} \mathbf{1}_{B(z, k(r+\rho))^{c}} \leq \phi(r, s)^{k} \leq \phi(r, t)^{k} .
$$

Covering the ball $B_{1}$ by a countable family of balls like $B\left(z, \frac{1}{4} r\right)$, we obtain that

$$
\sup _{0<s \leq t}\|u(s, \cdot)\|_{L^{\infty}\left(B_{1}\right)} \leq \phi(r, t)^{k} .
$$

Therefore, it follows from (3.5) and (3.2) that

$$
u(t, \cdot) \leq \phi(r, t)^{k+1} \text { in } \frac{1}{4} B
$$

proving (3.3).

Finally, in order to show (3.4), let $r:=R-\rho>0$. It follows from (3.3) that, for any $y \in M$ and $k \geq 1$,

$$
Q_{t} \mathbf{1}_{B(y, k R)^{c}} \leq \phi(R-\rho, t)^{k} \text { in } B(y, r / 4) .
$$

Fixing $x \in M$, using (3.6) for any $y \in B(x, R)$, and noticing that

$$
B(y, k R) \subset B(x,(k+1) R),
$$

we obtain

$$
Q_{t} \mathbf{1}_{B(x,(k+1) R)^{c}} \leq Q_{t} \mathbf{1}_{B(y, k R)^{c}} \leq \phi(R-\rho, t)^{k} \text { in } B(y, r / 4) .
$$

Covering $B(x, R)$ by a countable family of balls like $B(y, r / 4)$, we obtain

$$
Q_{t} \mathbf{1}_{B(x,(k+1) R)^{c}} \leq \phi(R-\rho, t)^{k},
$$

whence (3.8) follows by renaming $k$ to $k-1$.

Corollary 3.2. Assume that there exist constants $\varepsilon, \delta \in(0,1)$ such that, for all balls $B$ of radius $r$ and for all $t \in\left(0, T_{0}\right)$ where $T_{0} \in(0, \infty]$ provided that $t^{1 / \beta} \leq \delta(r \wedge \rho)$,

$$
1-Q_{t}^{B} \mathbf{1}_{B} \leq \varepsilon \text { in } \frac{1}{4} B
$$

Then, for any ball $B(x, r)$ and $t \in\left(0, T_{0}\right)$,

$$
Q_{t} \mathbf{1}_{B(x, r)^{c}} \leq C \exp \left(-c\left(\frac{r}{\rho} \wedge \frac{\rho}{t^{1 / \beta}}\right)\right) \text { in } B(x, \rho),
$$

where the constants $C, c>0$ depend only on $\varepsilon, \delta$.

Proof. If $r \leq 2 \rho$ or if $\delta \rho \leq t^{1 / \beta}$, then (3.8) is trivially satisfied by choosing a large enough constant $C$, because $Q_{t} \mathbf{1}_{B(x, r)^{c}} \leq 1$ and

$$
\frac{r}{\rho} \wedge \frac{\rho}{t^{1 / \beta}} \leq \max \left\{2, \delta^{-1}\right\}
$$

Assume that $\frac{r}{\rho}>2$ and $t^{1 / \beta}<\delta \rho$. Define the function $\phi(r, t)$ as follows:

$$
\phi(r, t)= \begin{cases}\varepsilon, & \text { if } t^{1 / \beta} \leq \delta \rho, \\ 1, & \text { otherwise. }\end{cases}
$$


Clearly, $\phi(r, t)$ is increasing in $t$, and (3.7) implies that (3.2) holds with this function $\phi$. Choose an integer $k \geq 1$ such that

$$
k<\frac{r}{2 \rho} \leq k+1 .
$$

Applying the inequality (3.4) of Theorem 3.1 with $R=2 \rho$ and noticing that $\phi(\rho, t)=\varepsilon$ because $t^{1 / \beta}<\delta \rho$, we obtain that in $B(x, 2 \rho)$,

$$
\begin{aligned}
Q_{t} \mathbf{1}_{B(x, r)^{c}} & \leq Q_{t} \mathbf{1}_{B(x, k R)^{c}} \leq \phi(\rho, t)^{k-1} \\
& =\varepsilon^{k-1}=\exp \left(-(k-1) \log \frac{1}{\varepsilon}\right) \\
& \leq \exp \left(-\left(\frac{r}{2 \rho}-2\right) \log \frac{1}{\varepsilon}\right)=C \exp \left(-c \frac{r}{\rho}\right),
\end{aligned}
$$

whence (3.8) follows.

\section{Heat semigroup of the truncated Dirichlet form}

Let $(\mathcal{E}, \mathcal{F})$ be a regular Dirichlet form in $L^{2}$ that allows a jump density $J(x, y)$ in the jump part. Fix $\rho \in(0, \infty)$ and define the bilinear form $\mathcal{E}^{(\rho)}(u, v)$ by

$$
\mathcal{E}^{(\rho)}(u, v)=\mathcal{E}^{(L)}(u, v)+\int_{M} \int_{B(x, \rho)}(u(x)-u(y))(v(x)-v(y)) J(x, y) d \mu(y) d \mu(x) .
$$

Clearly, the form $\mathcal{E}^{(\rho)}(u, v)$ is well defined for $u, v \in \mathcal{F}$ and

$$
\mathcal{E}^{(\rho)}(u) \leq \mathcal{E}(u) \text { for all } u \in \mathcal{F} \text {. }
$$

In this section, we prove that the form $\left(\mathcal{E}^{(\rho)}, \mathcal{F}\right)$ can be extended to a regular Dirichlet form $\left(\mathcal{E}^{(\rho)}, \mathcal{F}^{(\rho)}\right)$ and obtain upper estimates of the heat kernel $q_{t}$ of the truncated Dirichlet form $\left(\mathcal{E}^{(\rho)}, \mathcal{F}^{(\rho)}\right)$.

Proposition 4.1. For all $u \in \mathcal{F}$, we have

$$
\mathcal{E}(u) \leq \mathcal{E}^{(\rho)}(u)+4\|u\|_{2}^{2} \operatorname{esup}_{x \in M} \int_{B(x, \rho)^{c}} J(x, y) d \mu(y) .
$$

Proof. It follows from (1.4), (1.5) and (4.1) that

$$
\begin{aligned}
\mathcal{E}(u, u)-\mathcal{E}^{(\rho)}(u, u) & =\int_{M} \int_{B(x, \rho)^{c}}(u(x)-u(y))^{2} J(x, y) d \mu(y) d \mu(x) \\
& \leq 2 \int_{M} \int_{B(x, \rho)^{c}}\left(u(x)^{2}+u(y)^{2}\right) J(x, y) d \mu(y) d \mu(x) \\
& \leq 4 \int_{M} \int_{B(x, \rho)^{c}} u(x)^{2} J(x, y) d \mu(y) d \mu(x) \\
& \leq 4\|u\|_{2}^{2} \operatorname{esup}_{x \in M} \int_{B(x, \rho)^{c}} J(x, y) d \mu(y),
\end{aligned}
$$

which was to be proved.

Proposition 4.2. Assume that

$$
\operatorname{esup}_{x \in M} \int_{B(x, \rho)^{c}} J(x, y) d \mu(y)<\infty .
$$


Then the form $\left(\mathcal{E}^{(\rho)}, \mathcal{F}\right)$ is closable, and its closure $\left(\mathcal{E}^{(\rho)}, \mathcal{F}^{(\rho)}\right)$ in $L^{2}$ is a regular, $\rho$-local Dirichlet form in $L^{2}$.

Remark 4.3. Under the hypotheses $\left(V_{\leq}\right)$and $\left(J_{\leq}\right)$condition (4.4) is satisfied (see the proof of Proposition 4.7).

Proof. It follows easily from (4.1) that the form $\left(\mathcal{E}^{(\rho)}, \mathcal{F}\right)$ is symmetric, non-negative definite, and Markovian. In order to prove that it is closable, it suffices to show that if $\left\{u_{n}\right\}_{n=1}^{\infty}$ is a sequence from $\mathcal{F}$ such that

$$
u_{n} \stackrel{L^{2}}{\rightarrow} 0 \text { and } \mathcal{E}^{(\rho)}\left(u_{n}-u_{m}\right) \rightarrow 0 \text { as } n, m \rightarrow \infty,
$$

then $\mathcal{E}^{(\rho)}\left(u_{n}\right) \rightarrow 0$ as $n \rightarrow \infty$. Indeed, by (4.3) we have

$$
\mathcal{E}\left(u_{n}-u_{m}\right) \leq \mathcal{E}^{(\rho)}\left(u_{n}-u_{m}\right)+\text { const }\left\|u_{n}-u_{m}\right\|_{2}^{2} \rightarrow 0
$$

as $n, m \rightarrow \infty$. By the closedness of $(\mathcal{E}, \mathcal{F})$, we conclude that $\mathcal{E}\left(u_{n}\right) \rightarrow 0$, whence the claim follows from (4.2).

Let $\mathcal{F}^{(\rho)}$ be the domain of the closure of $\left(\mathcal{E}^{(\rho)}, \mathcal{F}\right)$. Then $\left(\mathcal{E}^{(\rho)}, \mathcal{F}^{(\rho)}\right)$ is a Dirichlet form. Let us prove that it is regular, that is, $\mathcal{F}^{(\rho)} \cap C_{0}$ is dense both in $C_{0}$ and $\mathcal{F}^{(\rho)}$. The former follows from the fact that $\mathcal{F} \cap C_{0}$ is dense in $C_{0}$ and $\mathcal{F} \subset \mathcal{F}^{(\rho)}$. To prove the latter, observe that, by construction of $\mathcal{F}^{(\rho)}$, for any $u \in \mathcal{F}^{(\rho)}$ and any $\varepsilon>0$ there exists $v \in \mathcal{F}$ such that

$$
\mathcal{E}^{(\rho)}(u-v)^{1 / 2}+\|u-v\|_{2}<\varepsilon .
$$

By the regularity of $(\mathcal{E}, \mathcal{F})$, there exists $f \in \mathcal{F} \cap C_{0}$, such that

$$
\mathcal{E}(v-f)^{1 / 2}+\|v-f\|_{2}<\varepsilon .
$$

Adding up these two inequalities, using (4.2) and the triangle inequalities, we obtain

$$
\mathcal{E}^{(\rho)}(u-f)^{1 / 2}+\|u-f\|_{2}<\text { const } \varepsilon,
$$

which proved the density of $\mathcal{F} \cap C_{0}$ in $\mathcal{F}^{(\rho)}$.

To show that $\left(\mathcal{E}^{(\rho)}, \mathcal{F}^{(\rho)}\right)$ is $\rho$-local, consider functions $u, v \in \mathcal{F}$ with compact supports and such that

$$
\operatorname{dist}(\operatorname{supp} u, \operatorname{supp} v)>\rho .
$$

If both points $x, y$ are outside one of the $\operatorname{supports} \operatorname{supp} u, \operatorname{supp} v$, then the integrand function in (4.1) vanishes. If they belong to different supports, then $d(x, y)>\rho$ so that the couple $(x, y)$ is outside the domain of integration in (4.1). Hence, the integral in (4.1) vanishes. Since $\mathcal{E}^{(L)}$ is local, we see that $\mathcal{E}^{(L)}(u, v)=0$, and hence $\mathcal{E}^{(\rho)}(u, v)=0$, which finishes the proof.

We now have two regular Dirichlet forms $(\mathcal{E}, \mathcal{F})$ and $\left(\mathcal{E}^{(\rho)}, \mathcal{F}^{(\rho)}\right)$. For any nonempty open subset $\Omega \subset M$, denote by $\left\{P_{t}^{\Omega}\right\}_{t>0}$ and $\left\{Q_{t}^{\Omega}\right\}_{t>0}$ the heat semigroups of $(\mathcal{E}, \mathcal{F}(\Omega))$ and $\left(\mathcal{E}^{(\rho)}, \mathcal{F}^{(\rho)}(\Omega)\right)$ respectively. Here we investigate the relationship between these two semigroups.

Proposition 4.4. For any non-empty open subset $\Omega$ of $M$ and any non-negative $f \in L^{2} \cap L^{1}(M, \mu)$, we have that, for all $t>0$ and almost all $x \in \Omega$,

$$
P_{t}^{\Omega} f(x) \leq Q_{t}^{\Omega} f(x)+2 t\|f\|_{1} \operatorname{esup}_{x \in M, y \in B(x, \rho)^{c}} J(x, y) .
$$


Proof. Without loss of generality we assume that $\Omega$ is precompact; otherwise we exhaust $\Omega$ by a sequence of precompact open subsets and then pass to the limit. It suffices to assume that $\|f\|_{1} \leq 1$. Note that the function

$$
v(t, \cdot)=Q_{t}^{\Omega} f
$$

is a weak solution of the heat equation associated with the form $\left(\mathcal{E}^{(\rho)}, \mathcal{F}^{(\rho)}(\Omega)\right)$, that is, for any $\psi \in \mathcal{F}^{(\rho)}(\Omega)$ and $t>0$,

$$
\left(\frac{\partial v}{\partial t}, \psi\right)+\mathcal{E}^{(\rho)}(v, \psi)=0
$$

(see Appendix 7 for details).

Let $\varphi$ be a cut-off function of the pair $(\Omega, M)$, that is, $\varphi \in \mathcal{F} \cap C_{0}, 0 \leq \varphi \leq 1$, and $\varphi \equiv 1$ in an open neighborhood of $\bar{\Omega}$. Consider the function

$$
u(t, x)=v(t, x)+2 w(\rho) t \varphi(x),
$$

where

$$
w(\rho):=\operatorname{esup}_{x \in M, y \in B(x, \rho)^{c}} J(x, y) .
$$

We claim that $u$ is a weak supersolution of the heat equation, associated with $(\mathcal{E}, \mathcal{F}(\Omega))$, that is,

$$
\left(\frac{\partial u}{\partial t}, \psi\right)+\mathcal{E}(u, \psi) \geq 0
$$

for all non-negative $\psi \in \mathcal{F}(\Omega)$ and $t>0$. Fix such a function $\psi$ and note that it satisfies (4.6) because $\mathcal{F}(\Omega) \subset \mathcal{F}^{(\rho)}(\Omega)$. Since $\varphi=1$ in $\Omega$, we see that $(\varphi, \psi)=\|\psi\|_{1}$ and

$$
\mathcal{E}(\varphi, \psi)=\lim _{t \rightarrow 0} t^{-1}\left(\varphi-P_{t} \varphi, \psi\right)=\lim _{t \rightarrow 0} t^{-1}\left(1-P_{t} \varphi, \psi\right) \geq 0 .
$$

Therefore, it follows from (4.7), (4.6) and (4.9) that

$$
\begin{aligned}
\left(\frac{\partial u}{\partial t}, \psi\right) & +\mathcal{E}(u, \psi)=\left(\frac{\partial v}{\partial t}+2 w(\rho) \varphi, \psi\right)+\mathcal{E}(v+2 w(\rho) t \varphi, \psi) \\
& =\left(\frac{\partial v}{\partial t}, \psi\right)+2 w(\rho)\|\psi\|_{1}+\mathcal{E}(v, \psi)+2 w(\rho) t \mathcal{E}(\varphi, \psi) \\
& \geq-\mathcal{E}^{(\rho)}(v, \psi)+2 w(\rho)\|\psi\|_{1}+\mathcal{E}(v, \psi) .
\end{aligned}
$$

On the other hand, using the facts that $v, \psi \geq 0$ and that

$$
\|v(t, \cdot)\|_{1}=\left\|Q_{t}^{\Omega} f\right\|_{1} \leq\|f\|_{1} \leq 1,
$$

we have that

$$
\begin{aligned}
\mathcal{E}(v, \psi) & -\mathcal{E}^{(\rho)}(v, \psi) \\
& =\int_{M} \int_{B(x, \rho)^{c}}(v(t, x)-v(t, y))(\psi(x)-\psi(y)) J(x, y) d \mu(y) d \mu(x) \\
& \geq-\int_{M} \int_{B(x, \rho)^{c}}(v(t, x) \psi(y)+v(t, y) \psi(x)) J(x, y) d \mu(y) d \mu(x) \\
& \geq-2 w(\rho) \int_{M} \psi(x) d \mu(x) \int_{B(x, \rho)^{c}} v(t, y) d \mu(y) \geq-2 w(\rho)\|\psi\|_{1} .
\end{aligned}
$$

Combining (4.10) and (4.11), we obtain (4.8). 
Finally, observing that

$$
u(t, \cdot) \stackrel{L^{2}(\Omega)}{\longrightarrow} f \text { as } t \rightarrow 0,
$$

we conclude that by the parabolic comparison principle

$$
u(t, \cdot) \geq P_{t}^{\Omega} f(\cdot)
$$

in $(0, \infty) \times \Omega$ (cf. [15, Lemma 4.16] or Lemma 7.3 in the Appendix). Substituting $u$ from (4.7) and using that $\varphi \equiv 1$ in $\Omega$, we obtain (4.5).

Corollary 4.5. For an open set $\Omega \subset M$, let $p_{t}^{\Omega}$ and $q_{t}^{\Omega}$ be the heat kernels of $(\mathcal{E}, \mathcal{F}(\Omega))$ and $\left(\mathcal{E}^{(\rho)}, \mathcal{F}^{(\rho)}(\Omega)\right)$, respectively. Then

$$
p_{t}^{\Omega}(x, y) \leq q_{t}^{\Omega}(x, y)+2 t \operatorname{esup}_{x \in M, y \in B(x, \rho)^{c}} J(x, y)
$$

for all $t>0$ and almost all $x, y \in \Omega$. In particular, for $\Omega=M$,

$$
p_{t}(x, y) \leq q_{t}(x, y)+2 t \operatorname{esup}_{x \in M, y \in B(x, \rho)^{c}} J(x, y)
$$

for all $t>0$ and almost all $x, y \in M$.

Proof. Inequality (4.12) immediately follows from (4.5).

Proposition 4.6. For any open subset $\Omega$ of $M$ and any non-negative $f \in L^{2} \cap$ $L^{\infty}(M, \mu)$, we have that, for all $t>0$ and almost all $x \in \Omega$,

$$
\left|P_{t}^{\Omega} f(x)-Q_{t}^{\Omega} f(x)\right| \leq 2 t\|f\|_{\infty} \operatorname{esup}_{x \in M} \int_{B(x, \rho)^{c}} J(x, y) d \mu(y) .
$$

Proof. The proof is similar to that of Proposition 4.4. It suffices to assume that $\|f\|_{\infty} \leq 1$. As in the proof of Proposition 4.4, set $v(t, \cdot)=Q_{t}^{\Omega} f$ and

$$
u(t, x)=v(t, x)+2 w(\rho) t \varphi(x),
$$

where $\varphi$ as before is a cut-off function of the pair $(\Omega, M)$, but $w(\rho)$ is now defined by

$$
w(\rho):=\operatorname{esup}_{x \in M} \int_{B(x, \rho)^{c}} J(x, y) d \mu(y) .
$$

Then $u$ satisfies (4.8) for all non-negative $\psi \in \mathcal{F}(\Omega)$ and $t>0$, because similar to (4.11) we have

$$
\begin{aligned}
\mathcal{E}(v, \psi) & -\mathcal{E}^{(\rho)}(v, \psi) \\
& \geq-\int_{M} \int_{B(x, \rho)^{c}}(v(t, x) \psi(y)+v(t, y) \psi(x)) J(x, y) d \mu(y) d \mu(x) \\
& \geq-2\|v(t, \cdot)\|_{\infty} \int_{M} \psi(x) d \mu(x) \int_{B(x, \rho)^{c}} J(x, y) d \mu(y) \\
& \geq-2 w(\rho)\|\psi\|_{1},
\end{aligned}
$$

where we have used that

$$
\|v(t, \cdot)\|_{\infty}=\left\|Q_{t}^{\Omega} f\right\|_{\infty} \leq\|f\|_{\infty} \leq 1
$$

Repeating the end of the proof of Proposition 4.4 we obtain that, for all $t>0$ and almost all $x \in \Omega$,

$$
P_{t}^{\Omega} f(x) \leq Q_{t}^{\Omega} f(x)+2 w(\rho) t
$$


In order to show the inequality

$$
P_{t}^{\Omega} f(x) \geq Q_{t}^{\Omega} f(x)-2 w(\rho) t,
$$

we instead consider the function

$$
u=P_{t}^{\Omega} f(x)+2 w(\rho) t \varphi .
$$

In a similar way, one can verify that $u$ is a supersolution of (4.6). Hence, by the comparison principle of Lemma 7.3. we obtain that $u \geq Q_{t}^{\Omega} f$ in $(0, \infty) \times \Omega$, thus finishing the proof.

Next we show the existence of the heat kernel $q_{t}$ of $\left(\mathcal{E}^{(\rho)}, \mathcal{F}^{(\rho)}\right)$ and derive its on-diagonal upper bound.

Proposition 4.7. Let $(\mathcal{E}, \mathcal{F})$ be a regular Dirichlet form in $L^{2}$ with a jump density $J(x, y)$ and let $\left(\mathcal{E}^{(\rho)}, \mathcal{F}^{(\rho)}\right)$ be the truncated form as in (4.1). If $(\mathcal{E}, \mathcal{F})$ satisfies the conditions $(D U E),\left(V_{\leq}\right)$and $\left(J_{\leq}\right)$, then the heat kernel $q_{t}$ of $\left(\mathcal{E}^{(\rho)}, \mathcal{F}^{(\rho)}\right)$ exists and satisfies the on-diagonal upper estimate

$$
q_{t}(x, y) \leq \frac{C}{t^{\alpha / \beta}} \exp \left(4 \rho^{-\beta} t\right)
$$

for all $t>0$ and $\mu$-almost all $x, y \in M$, where $C>0$ depends on the constants in the hypotheses but is independent of $\rho$.

Proof. First observe that $(D U E)$ implies the Nash inequality:

$$
\|u\|_{2}^{2\left(1+\frac{2 \beta}{\alpha}\right)} \leq C \mathcal{E}(u)
$$

for all $u \in \mathcal{F} \cap L^{1}$ with $\|u\|_{1}=1$, for $C>0$ (cf. [8, Theorem (2.1)]). By conditions $\left(V_{\leq}\right)$and $\left(J_{\leq}\right)$, we have that

$$
\begin{aligned}
\int_{B(x, \rho)^{c}} J(x, y) d \mu(y) & \leq C \int_{B(x, \rho)^{c}} d(x, y)^{-(\alpha+\beta)} d \mu(y) \\
& =C \int_{\rho}^{\infty} s^{-(\alpha+\beta)} d V(x, s) \\
& \leq c \rho^{-\beta},
\end{aligned}
$$

and hence there exists $c>0$ independent of $\rho$ such that

$$
w(\rho):=\operatorname{esup}_{x \in M} \int_{B(x, \rho)^{c}} J(x, y) d \mu(y) \leq c \rho^{-\beta} .
$$

Therefore, we obtain from (4.20) and (4.3) that for all $u \in \mathcal{F}^{(\rho)} \cap L^{1}$ with $\|u\|_{1}=1$,

$$
\|u\|_{2}^{2\left(1+\frac{2 \beta}{\alpha}\right)} \leq C\left(\mathcal{E}^{(\rho)}(u)+4 \rho^{-\beta}\|u\|_{2}^{2}\right) .
$$

Hence, by [8, Theorem $(2.1)]$, the heat kernel $q_{t}$ of $\left(\mathcal{E}^{(\rho)}, \mathcal{F}^{(\rho)}\right)$ exists and satisfies (4.19).

The following proposition gives a survival estimate for the Dirichlet heat semigroup $\left\{Q_{t}^{B}\right\}_{t>0}$ associated with $\left(\mathcal{E}^{(\rho)}, \mathcal{F}^{(\rho)}\right)$ for any ball $B$. 
Proposition 4.8. If conditions $(S),\left(J_{\leq}\right)$and $\left(V_{\leq}\right)$are satisfied, then we have that, for any $\varepsilon^{\prime} \in(\varepsilon, 1)$, there exists $\delta^{\prime}>0$ independent of $\rho$ such that, for all $t>0$ and all balls $B$ of radius $r$,

$$
1-Q_{t}^{B} \mathbf{1}_{B}(x) \leq \varepsilon^{\prime} \text { for } \mu \text {-almost all } x \in \frac{1}{4} B,
$$

provided that $t^{1 / \beta} \leq \delta^{\prime}(r \wedge \rho)$.

Proof. We show (4.22) by using Proposition 4.6. Indeed, let $B=B\left(x_{0}, r\right)$ and choose $\Omega=B$ and $f=\mathbf{1}_{B}$ in (4.14). It follows, using (4.21), that for all $t>0$ and almost all $x \in B$,

$$
P_{t}^{B} \mathbf{1}_{B}(x) \leq Q_{t}^{B} \mathbf{1}_{B}(x)+2 C \rho^{-\beta} t\left\|\mathbf{1}_{B}\right\|_{\infty}=Q_{t}^{B} \mathbf{1}_{B}(x)+c \rho^{-\beta} t .
$$

Hence, we have from condition $(S)$ that

$$
\begin{aligned}
1-Q_{t}^{B} \mathbf{1}_{B}(x) & \leq 1-P_{t}^{B} \mathbf{1}_{B}(x)+c \rho^{-\beta} t \\
& \leq \varepsilon+c \rho^{-\beta} t \leq \varepsilon^{\prime} \text { in } \frac{1}{4} B
\end{aligned}
$$

if $t^{1 / \beta} \leq \delta r$ and if $c \rho^{-\beta} t \leq \varepsilon^{\prime}-\varepsilon$, for any $\varepsilon^{\prime} \in(\varepsilon, 1)$. This finishes the proof.

The following theorem gives the upper estimate of the heat kernel $q_{t}$ of $\left(\mathcal{E}^{(\rho)}, \mathcal{F}^{(\rho)}\right)$.

Theorem 4.9. Let $\left(\mathcal{E}^{(\rho)}, \mathcal{F}^{(\rho)}\right)$ be the truncated Dirichlet form as above. If $(\mathcal{E}, \mathcal{F})$ satisfies the conditions $(D U E),\left(J_{\leq}\right),(S)$ and $\left(V_{\leq}\right)$, then, for all $t>0$ and $\mu$-almost all $x, y \in M$,

$$
q_{t}(x, y) \leq \frac{C}{t^{\alpha / \beta}} \exp \left(4 \rho^{-\beta} t\right) \exp \left(-c\left(\frac{d(x, y)}{\rho} \wedge \frac{\rho}{t^{1 / \beta}}\right)\right),
$$

where the constants $C, c>0$ depend on the constants in the hypotheses but are independent of $\rho$.

Proof. Fix $x_{0}, y_{0} \in M$ and $t>0$. Set $r=\frac{1}{2} d\left(x_{0}, y_{0}\right)$. By the semigroup property, we have that

$$
\begin{aligned}
q_{2 t}(x, y)= & \int_{M} q_{t}(x, z) q_{t}(z, y) d \mu(z) \\
\leq & \int_{B\left(x_{0}, r\right)^{c}} q_{t}(x, z) q_{t}(z, y) d \mu(z) \\
& +\int_{B\left(y_{0}, r\right)^{c}} q_{t}(x, z) q_{t}(z, y) d \mu(z) .
\end{aligned}
$$

Using (4.19) and (3.8) with $T_{0}=\infty$, we obtain that

$$
\begin{aligned}
\int_{B\left(x_{0}, r\right)^{c}} q_{t}(x, z) q_{t}(z, y) d \mu(z) & \leq \frac{C}{t^{\alpha / \beta}} \exp \left(4 \rho^{-\beta} t\right) \int_{B\left(x_{0}, r\right)^{c}} q_{t}(x, z) d \mu(z) \\
& \leq \frac{C}{t^{\alpha / \beta}} \exp \left(4 \rho^{-\beta} t\right) \exp \left(-c\left(\frac{r}{\rho} \wedge \frac{\rho}{t^{1 / \beta}}\right)\right)
\end{aligned}
$$

for $\mu$-almost all $x \in B\left(x_{0}, \rho\right)$ and $y \in M$. Similarly,

$$
\int_{B\left(y_{0}, r\right)^{c}} q_{t}(x, z) q_{t}(z, y) d \mu(z) \leq \frac{C}{t^{\alpha / \beta}} \exp \left(4 \rho^{-\beta} t\right) \exp \left(-c\left(\frac{r}{\rho} \wedge \frac{\rho}{t^{1 / \beta}}\right)\right)
$$

for $\mu$-almost all $y \in B\left(y_{0}, \rho\right)$ and $x \in M$. Hence, inequality (4.24) follows. 


\section{Proof of Theorem 2.1}

In this section, we prove Theorem 2.1. The proof of the main implication (2.10) will be done in two steps. In the first step we show that, for any real $n \geq 0$,

$$
p_{t}(x, y) \leq \frac{c(n)}{t^{\alpha / \beta}}\left(1+\frac{d(x, y)}{t^{1 / \beta}}\right)^{-\frac{(\alpha+\beta) n}{n+\alpha+\beta}}
$$

for almost all $x, y \in M$ and all $t>0$. In the second step, we obtain the desired estimate $(U E)$ by a self-improvement of (5.1).

Lemma 5.1. Assume that all the hypotheses of Theorem 2.1 hold. Then

$$
\left(V_{\leq}\right)+(D U E)+\left(J_{\leq}\right)+(S) \Rightarrow \text { (5.1). }
$$

Proof. Since the estimate (5.1) becomes stronger for larger $n$, it suffices to assume that $n \geq \alpha+\beta$. Fix some $t>0, x, y \in M$ and set $r=d(x, y)$. If $r<t^{1 / \beta}$, then (5.1) follows from $(D U E)$. Hence, we assume in the sequel that

$$
r \geq t^{1 / \beta} \text {. }
$$

Let $\rho>0$ be such that

$$
\frac{\rho}{t^{1 / \beta}} \geq 1 \text { and } \frac{\rho}{t^{1 / \beta}} \geq \frac{r}{\rho}
$$

(we specify the value of $\rho$ below). Using Theorem 4.9 and the elementary inequality

$$
\exp (-c s) \leq c_{1}(n) s^{-n} \text { for all } s>0,
$$

we obtain from (4.24) and (5.3) that

$$
q_{t}(x, y) \leq \frac{C_{1}}{t^{\alpha / \beta}} \exp \left(-c_{1} \frac{r}{\rho}\right) \leq \frac{C(n)}{t^{\alpha / \beta}}\left(\frac{r}{\rho}\right)^{-n} .
$$

Therefore, it follows from (4.13) and $\left(J_{\leq}\right)$that

$$
\begin{aligned}
p_{t}(x, y) & \leq q_{t}(x, y)+c \rho^{-(\alpha+\beta)} t \\
& \leq \frac{C(n)}{t^{\alpha / \beta}}\left[\left(\frac{\rho}{r}\right)^{n}+\rho^{-(\alpha+\beta)} t^{1+\alpha / \beta}\right] .
\end{aligned}
$$

Now choose $\rho$ such that

$$
\left(\frac{\rho}{r}\right)^{n}=\rho^{-(\alpha+\beta)} t^{1+\alpha / \beta},
$$

that is,

$$
\rho=r^{\frac{n}{n+\alpha+\beta}}\left(t^{1 / \beta}\right)^{\frac{\alpha+\beta}{n+\alpha+\beta}} .
$$

This $\rho$ satisfies (5.3), since by (5.2) and $n \geq \alpha+\beta$,

$$
\begin{aligned}
\frac{\rho}{t^{1 / \beta}} & =\left(\frac{r}{t^{1 / \beta}}\right)^{\frac{n}{n+\alpha+\beta}} \geq 1, \\
\frac{\rho}{t^{1 / \beta}} & =\left(\frac{r}{t^{1 / \beta}}\right)^{\frac{n}{n+\alpha+\beta}} \geq\left(\frac{r}{t^{1 / \beta}}\right)^{\frac{\alpha+\beta}{n+\alpha+\beta}}=\frac{r}{\rho} .
\end{aligned}
$$

Substituting the value of $\rho$ to (5.4), we obtain

$$
p_{t}(x, y) \leq \frac{2 C(n)}{t^{\alpha / \beta}}\left(\frac{\rho}{r}\right)^{n}=\frac{c(n)}{t^{\alpha / \beta}}\left(\frac{r}{t^{1 / \beta}}\right)^{-\frac{(\alpha+\beta) n}{n+\alpha+\beta}},
$$

which finishes the proof. 
As a second step, we now prove Theorem 2.1

Proof of Theorem 2.1. To show (2.10), we divide the proof into four steps.

Step 1. For all $r>0$ and $\mu$-almost all $x$, it follows from (5.1) (cf. [17, Inequality (3.7), p. 2072]) that

$$
\begin{aligned}
\int_{B(x, r)^{c}} p_{t}(x, y) d \mu(y) & \leq \int_{B(x, r)^{c}} \frac{c(n)}{t^{\alpha / \beta}}\left(1+\frac{d(x, y)}{t^{1 / \beta}}\right)^{-\frac{(\alpha+\beta) n}{n+\alpha+\beta}} d \mu(y) \\
& \leq c(n) \int_{\frac{1}{2} r t^{-1 / \beta}}^{\infty} s^{\alpha-1}(1+s)^{-\frac{(\alpha+\beta) n}{n+\alpha+\beta}} d s \\
& \leq C(n)\left(r t^{-1 / \beta}\right)^{-\theta},
\end{aligned}
$$

where

$$
\theta=\frac{n \beta-\alpha(\alpha+\beta)}{n+\alpha+\beta} \in(0, \beta)
$$

provided that

$$
n>\alpha(1+\alpha / \beta) .
$$

Step 2. Let $B$ be any ball of radius $r$. Using [18, Lemma 6.1], we obtain from (5.6) that there is $c>0$ such that

$$
1-P_{t}^{B} \mathbf{1}_{B}(x) \leq c\left(r t^{-1 / \beta}\right)^{-\theta} \quad \text { for } \mu \text {-a.a. } x \in \frac{1}{4} B .
$$

Note that the conservativeness of $(\mathcal{E}, \mathcal{F})$ is required in [18, Lemma 6.1]. Therefore, using (4.23),

$$
1-Q_{t}^{B} \mathbf{1}_{B}(x) \leq c_{1}\left[\left(r t^{-1 / \beta}\right)^{-\theta}+\rho^{-\beta} t\right]:=\phi(r, t)
$$

for all $t>0$ and $\mu$-almost all $x \in \frac{1}{4} B$, where $c_{1}>0$ is independent of the ball $B$ and $\rho$.

Step 3. We prove the following improvement of estimate (4.24): for all $t>0, k \geq 1$, and all $x_{0}, y_{0} \in M$ with $d\left(x_{0}, y_{0}\right)>4 k \rho$,

$$
q_{t}(x, y) \leq \frac{C(k)}{t^{\alpha / \beta}} \exp \left(4 \rho^{-\beta} t\right)\left(1+\rho t^{-1 / \beta}\right)^{-\theta(k-1)},
$$

for $\mu$-almost all $x \in B\left(x_{0}, \rho\right), y \in B\left(y_{0}, \rho\right)$, where the constant $C(k)$ is independent of $\rho$. The argument is similar to that of Theorem 4.9. but we use the sharper estimate (5.10) instead of survival estimate $(S)$. Indeed, fix $k \geq 1, t>0$ and fix $x_{0}, y_{0} \in M$. Set

$$
r=\frac{1}{2} d\left(x_{0}, y_{0}\right)>2 k \rho \text {. }
$$

Assume that

$$
\rho t^{-1 / \beta}>1
$$

otherwise (5.11) follows directly from (4.19). 
Applying inequality (3.4) in Theorem 3.1 with $R=2 \rho$ and with function $\phi(r, t)$ from (5.10), we obtain that in $B\left(x_{0}, \rho\right)$,

$$
\begin{aligned}
Q_{t} \mathbf{1}_{B\left(x_{0}, r\right)^{c}} & \leq Q_{t} \mathbf{1}_{B\left(x_{0}, 2 k \rho\right)^{c}} \leq \phi(\rho, t)^{k-1} \\
& =c_{1}^{k-1}\left[\left(\rho t^{-1 / \beta}\right)^{-\theta}+\rho^{-\beta} t\right]^{k-1} \\
& \leq C(k)\left(\rho t^{-1 / \beta}\right)^{-\theta(k-1)}
\end{aligned}
$$

since

$$
\rho^{-\beta} t=\left(\rho t^{-1 / \beta}\right)^{-\beta} \leq\left(\rho t^{-1 / \beta}\right)^{-\theta}
$$

Similarly, we have that in $B\left(y_{0}, \rho\right)$,

$$
Q_{t} \mathbf{1}_{B\left(y_{0}, r\right)^{c}} \leq C(k)\left(\rho t^{-1 / \beta}\right)^{-\theta(k-1)} .
$$

Therefore, it follows from (4.25) and (4.19) that

$$
\begin{aligned}
q_{2 t}(x, y) & \leq\left\|q_{t}(\cdot, y)\right\|_{L^{\infty}(M)} Q_{t} \mathbf{1}_{B\left(x_{0}, r\right)^{c}}(x)+\left\|q_{t}(x, \cdot)\right\|_{L^{\infty}(M)} Q_{t} \mathbf{1}_{B\left(y_{0}, r\right)^{c}}(y) \\
& \leq \frac{C(k)}{t^{\alpha / \beta}} \exp \left(4 \rho^{-\beta} t\right)\left(\rho t^{-1 / \beta}\right)^{-\theta(k-1)}
\end{aligned}
$$

for all $t>0$ and $\mu$-almost all $x \in B\left(x_{0}, \rho\right), y \in B\left(y_{0}, \rho\right)$, thus proving (5.11).

Step 4. Finally, we prove $(U E)$. Fix $x_{0}, y_{0} \in M$, and let $r=\frac{1}{2} d\left(x_{0}, y_{0}\right)$ as in the above step. We show that

$$
p_{t}(x, y) \leq \frac{c}{t^{\alpha / \beta}}\left(1+\frac{r}{t^{1 / \beta}}\right)^{-(\alpha+\beta)}
$$

for all $t>0$ and $\mu$-almost all $x \in B\left(x_{0}, \rho\right), y \in B\left(y_{0}, \rho\right)$, for all small enough $\rho>0$. Indeed, it suffices to assume that $r t^{-1 / \beta}$ is sufficiently large. Fix an integer $n$ such that (5.8) holds, and hence the number $\theta$ from (5.7) is also fixed. Let

$$
k=2+[(\alpha+\beta) / \theta]
$$

so that

$$
\theta(k-1) \geq \alpha+\beta
$$

and let $\rho=\frac{r}{8 k}$. Then

$$
\rho^{-\beta} t=\left(\frac{r t^{-1 / \beta}}{8 k}\right)^{-\beta} \leq C
$$

and hence, using (4.13) again and (5.11),

$$
\begin{aligned}
p_{t}(x, y) & \leq q_{t}(x, y)+c \rho^{-(\alpha+\beta)} t \\
& \leq \frac{C}{t^{\alpha / \beta}}\left(1+\frac{r}{8 k t^{1 / \beta}}\right)^{-\theta(k-1)}+c\left(\frac{r}{8 k}\right)^{-(\alpha+\beta)} t \\
& \leq \frac{c}{t^{\alpha / \beta}}\left(\frac{r}{t^{1 / \beta}}\right)^{-(\alpha+\beta)}
\end{aligned}
$$

for all $t>0$ and $\mu$-almost all $x \in B\left(x_{0}, \rho\right), y \in B\left(y_{0}, \rho\right)$, thus proving (5.12). Therefore, $(U E)$ follows. 


\section{Heat kernel bounds using EFFective REsistance}

In this section we show how Theorem 2.3 can be applied for a certain class of metric measure spaces with effective resistance.

Let $(\mathcal{E}, \mathcal{F})$ be a regular Dirichlet form in $L^{2}(M, \mu)$ as before. Recall that the effective resistance $R(A, B)$ between two disjoint non-empty closed subsets $A$ and $B$ of $M$ is defined by

$$
R(A, B)^{-1}=\inf \left\{\mathcal{E}(u): u \in \mathcal{F} \cap C_{0},\left.u\right|_{A}=1 \text { and }\left.u\right|_{B}=0\right\} .
$$

It follows from (6.1) that, for any fixed $A, R(A, B)$ is a non-increasing function of $B$. Denote

$$
R(x, B):=R(\{x\}, B) \text { and } R(x, y):=R(\{x\},\{y\}) .
$$

In general, it may happen that $R(x, y)=\infty$ for some points $x, y \in M$. In this section we will exclude this case. We assume that the following two conditions hold:

$\left(\mathbf{R}_{1}\right)$ : There exist constants $C, \gamma>0$ such that

$$
|u(x)-u(y)|^{2} \leq C d(x, y)^{\gamma} \mathcal{E}(u)
$$

for all $u \in \mathcal{F} \cap C_{0}$ and all $x, y \in M$.

$\left(\mathbf{R}_{2}\right)$ : There exist constants $C, \gamma>0$ such that, for all balls $B=B(x, r)$,

$$
R\left(x, B(x, r)^{c}\right) \geq C^{-1} r^{\gamma} .
$$

Observe that condition $\left(R_{1}\right)$ is the Morrey-Sobolev type inequality in the framework of Dirichlet forms. As we will see, it implies that all functions in $\mathcal{F}$ are Hölder continuous.

Consider also the following estimates of the effective resistance:

$\left(\mathbf{R}_{\leq}\right)$: There exist constants $C, \gamma>0$ such that, for all $x, y \in M$,

$$
R(x, y) \leq C d(x, y)^{\gamma} .
$$

$\left(\mathbf{R}_{\geq}\right)$: There exist constants $C, \gamma>0$ such that, for all $x, y \in M$,

$$
R(x, y) \geq C^{-1} d(x, y)^{\gamma} .
$$

Applying the definition of (6.1) to $A=\{x\}$ and $B=\{y\}$ we see that

$$
\left(R_{1}\right) \Rightarrow\left(R_{\leq}\right) .
$$

Observe that also

$$
\left(R_{2}\right) \Rightarrow\left(R_{\geq}\right) .
$$

Indeed, for any two points $x, y \in M$, taking $r=d(x, y)$ in $\underline{6.3}$ ) and noticing that $y \in B(x, r)^{c}$, we obtain

$$
R(x, y) \geq R\left(x, B(x, r)^{c}\right) \geq C^{-1} r^{\gamma}=C^{-1} d(x, y)^{\gamma} .
$$

In the next subsections, we will investigate various sufficient conditions for $\left(R_{1}\right)$ and $\left(R_{2}\right)$, as well as their consequences. Then we will prove Theorem 6.13 below that states the equivalence $(U E) \Leftrightarrow\left(J_{\leq}\right)$under the standing hypotheses $\left(R_{1}\right)$ and $\left(R_{2}\right)$. 


\subsection{Condition $\left(R_{1}\right)$.}

Lemma 6.1. If $\left(R_{1}\right)$ and $(V \geq)$ are satisfied, then any function $u \in \mathcal{F}$ admits a continuous version that satisfies (6.2). Consequently, all functions in $\mathcal{F}$ have Hölder continuous versions with the Hölder exponent $\gamma / 2$.

Proof. By the regularity of $(\mathcal{E}, \mathcal{F})$, for every $u \in \mathcal{F}$ there exists a sequence $\left\{u_{n}\right\}_{n=1}^{\infty}$ $\subset \mathcal{F} \cap C_{0}$ such that $\left\|u_{n}-u\right\|_{2} \rightarrow 0$ and $\mathcal{E}\left(u-u_{n}\right) \rightarrow 0$ as $n \rightarrow \infty$. By $\left(R_{1}\right)$ we have

$$
\left|u_{n}(y)-u_{n}(x)\right|^{2} \leq C d(x, y)^{\gamma} \mathcal{E}\left(u_{n}\right)
$$

for all $x, y \in M$ and $n \geq 1$. Since the sequence $\left\{\mathcal{E}\left(u_{n}\right)\right\}$ is uniformly bounded, it follows from (6.6) that the sequence $\left\{u_{n}\right\}_{n=1}^{\infty}$ is equicontinuous in $(M, d)$. Let us prove that the sequence $\left\{u_{n}\right\}_{n=1}^{\infty}$ is uniformly bounded in $M$ in sup-norm. Indeed, it follows from (6.6) that

$$
\left|u_{n}(y)-u_{n}(x)\right| \leq c d(x, y)^{\gamma / 2},
$$

with some $c>0$, and hence

$$
u_{n}(x)^{2} \geq\left(u_{n}(y)-c d(x, y)^{\gamma / 2}\right)_{+}^{2} .
$$

Integrating this inequality with respect to $x \in M$ with a fixed $y$ and setting $m=$ $\left|u_{n}(y)\right|$, we obtain

$$
\left\|u_{n}\right\|_{2}^{2} \geq \int_{M}\left(m-c d(x, y)^{\gamma / 2}\right)_{+}^{2} d \mu(x) \geq \int_{0}^{R}\left(m-c s^{\gamma / 2}\right)^{2} d V(x, s),
$$

where $R>0$ is chosen so that $\frac{1}{2} m=c R^{\gamma / 2}$. It follows that $m-c s^{\gamma / 2} \geq \frac{1}{2} m$ and

$$
\left\|u_{n}\right\|_{2}^{2} \geq \int_{0}^{R} \frac{1}{4} m^{2} d V(x, s)=\frac{1}{4} m^{2} V(x, R) \geq c_{1} m^{2} R^{\alpha}=c_{2} m^{2+2 \frac{\gamma}{\alpha}},
$$

where we have also used $(V \geq)$. It follows that

$$
\left|u_{n}(y)\right| \leq C\left\|u_{n}\right\|_{2}^{\frac{\alpha}{\alpha+\gamma}} .
$$

Since the sequence $\left\{\left\|u_{n}\right\|_{2}\right\}$ is uniformly bounded and the constant $C$ is independent of $n$ and $y$, we conclude that the sequence $\left\{\sup \left|u_{n}\right|\right\}$ is uniformly bounded as well.

By the Arzela-Ascoli theorem, the sequence $\left\{u_{n}\right\}$ has a subsequence that converges locally uniformly to a continuous function $\widetilde{u}$, that is, a continuous version of $u$. Obviously, $\widetilde{u}$ satisfies (6.2) that proves the Hölder continuity.

The following proposition is useful for verifying $\left(R_{1}\right)$.

Proposition 6.2. Let $(\mathcal{E}, \mathcal{F})$ be a regular Dirichlet form $(\mathcal{E}, \mathcal{F})$ in $L^{2}(M, \mu)$. If $(V)$ and $\left(J_{\geq}\right)$are satisfied with $0<\alpha<\beta$, then $\left(R_{1}\right)$ holds with $\gamma=\beta-\alpha$. Consequently, all functions in $\mathcal{F}$ are Hölder continuous with the Hölder exponent $\frac{1}{2}(\beta-\alpha)$.

Proof. We use in the proof the following fact: if $\mu$ is $\alpha$-regular and $0<\alpha<\beta$, then there exists a constant $C>0$ such that for all functions $u \in L^{2}(M, \mu)$ the following inequality holds:

$$
|u(y)-u(x)|^{2} \leq C d(x, y)^{\beta-\alpha} \sup _{0<r<r_{0}} \int_{M} \int_{B(x, r)} \frac{1}{r^{\alpha+\beta}}|u(y)-u(x)|^{2} d \mu(y) d \mu(x)
$$


for all Lebesgue points $x, y \in M$ of $u$ with $d(x, y)<r_{0} / 3$, where $r_{0} \in(0,+\infty]$ is fixed but arbitrary (cf. [17, the proof of Theorem 4.11(iii)]).

Since by hypothesis $\mu$ is $\alpha$-regular and $J(x, y) \geq c d(x, y)^{-(\alpha+\beta)}$, we have, for all $u \in \mathcal{F} \cap C_{0}$ and for all $r>0$,

$$
\begin{aligned}
\mathcal{E}^{(J)}(u) & =\int_{M \times M} \int_{M}|u(y)-u(x)|^{2} J(x, y) d \mu(y) d \mu(x) \\
& \geq c \int_{M \times M} \int_{\times}|u(y)-u(x)|^{2} d(x, y)^{-(\alpha+\beta)} d \mu(y) d \mu(x) \\
& \geq c \int_{M} \int_{B(x, r)} \frac{1}{r^{\alpha+\beta}}|u(y)-u(x)|^{2} d \mu(y) d \mu(x) .
\end{aligned}
$$

Combining this with (6.7), we obtain

$$
|u(y)-u(x)|^{2} \leq C d(x, y)^{\beta-\alpha} \mathcal{E}(u),
$$

which yields $\left(R_{1}\right)$ with $\gamma=\beta-\alpha$.

The second claim follows from Lemma 6.1

6.2. Parabolicity. Condition $\left(R_{1}\right)$ can also be obtained from $\left(R_{\leq}\right)$assuming in addition the parabolicity of $(\mathcal{E}, \mathcal{F})$.

For any precompact set $K$ define its capacity by

$$
\operatorname{cap}(K)=\inf \left\{\mathcal{E}(u): u \in \mathcal{F} \cap C_{0},\left.u\right|_{K}=1\right\},
$$

that is, $\operatorname{cap}(K)=R(K,\{\infty\})^{-1}$. Obviously, $\operatorname{cap}(K)$ is a monotone increasing function of $K$.

Definition 6.3. A Dirichlet form $(\mathcal{E}, \mathcal{F})$ is called parabolid 1 if $\operatorname{cap}(K)=0$ for any compact subset of $M$.

A trivial sufficient condition for the parabolicity is that $1 \in \mathcal{F}$ and $\mathcal{E}(1,1)=0$. Indeed, in this case the constant function 1 can be used as a test function $u$ in (6.9) that yields $\operatorname{cap}(K)=0$.

Lemma 6.4. Let $(\mathcal{E}, \mathcal{F})$ be a regular Dirichlet form in $L^{2}$. Then the following statements hold:

$$
\text { parabolicity } \Rightarrow \text { conservativeness. }
$$

Proof. Indeed, if $(\mathcal{E}, \mathcal{F})$ is parabolic, then there exists a sequence of functions $\left\{\varphi_{n}\right\}_{n=1}^{\infty}$ from $\mathcal{F}$ such that $0 \leq \varphi_{n} \uparrow 1$ and

$$
\mathcal{E}\left(\varphi_{n}\right) \rightarrow 0
$$

as $n \uparrow \infty$. In particular, we have that

$$
\mathcal{E}\left(\varphi_{n}, v\right) \rightarrow 0
$$

as $n \uparrow \infty$, for any $v \in \mathcal{F} \cap L^{1}$. Hence, $(\mathcal{E}, \mathcal{F})$ is conservative (cf. 11, Theorem 1.6.6, p. 63]).

\footnotetext{
${ }^{1}$ The term "parabolic" comes from classification theory of Riemann surfaces. A simply connected non-compact Riemann surface is conformally equivalent either to the Euclidean plane $\mathbb{R}^{2}$ or to the hyperbolic plane $\mathbb{H}^{2}$. In the first case the surface is called parabolic, whereas in the second case - hyperbolic. It is known that the parabolicity of the Riemann surface is equivalent to the vanishing of the capacity of any compact subset (cf. [12]).
} 
The following gives a criterion of verifying the parabolicity.

Proposition 6.5. Let $(\mathcal{E}, \mathcal{F})$ be a Dirichlet form in $L^{2}(M, \mu)$ and let $(M, d)$ be unbounded. Then the following implication holds:

$$
\left(R_{1}\right)+\left(R_{\geq}\right) \Rightarrow \text { parabolicity of }(\mathcal{E}, \mathcal{F}) .
$$

Proof. Let $K$ be a compact subset of $M$ and fix $x_{0} \in K$. Fix a number $\eta \in(0,1)$ to be determined later on, and choose a point $x \notin K$ such that $K \subset B\left(x_{0}, \eta r\right)$ where $r=d\left(x_{0}, x\right)$. Let $\psi_{x} \in \mathcal{F}$ such that $\psi_{x}\left(x_{0}\right)=1, \psi_{x}(x)=0,0 \leq \psi_{x} \leq 1$ in $M$, and

$$
\mathcal{E}\left(\psi_{x}\right)=R\left(x_{0}, x\right)^{-1} .
$$

It follows from $\left(R_{1}\right),\left(R_{\geq}\right)$that, for all $y \in B\left(x_{0}, \eta r\right)$,

$$
\begin{aligned}
\left(1-\psi_{x}(y)\right)^{2} & =\left(\psi_{x}\left(x_{0}\right)-\psi_{x}(y)\right)^{2} \leq C d\left(x_{0}, y\right)^{\gamma} \mathcal{E}\left(\psi_{x}\right) \\
& \leq C \frac{(\eta r)^{\gamma}}{R\left(x_{0}, x\right)} \leq c \eta^{\gamma} .
\end{aligned}
$$

Now choose $\eta$ so small that $c \eta^{\gamma} \leq \frac{1}{4}$. Hence, we have $\psi_{x} \geq \frac{1}{2}$ in $B\left(x_{0}, \eta r\right) \supset K$. It follows that $f:=2 \psi_{x} \geq 1$ on $K$, and

$$
\begin{aligned}
\operatorname{cap}(K) & \leq \mathcal{E}(f)=4 \mathcal{E}\left(\psi_{x}\right) \\
& =4 R\left(x_{0}, x\right)^{-1} \leq C r^{-\gamma}
\end{aligned}
$$

by using condition $\left(R_{\geq}\right)$. Letting $r=d\left(x_{0}, x\right) \rightarrow \infty$, we obtain that $\operatorname{cap}(K)=0$, showing that $(\mathcal{E}, \mathcal{F})$ is parabolic.

Proposition 6.6. Assume that $(\mathcal{E}, \mathcal{F})$ is parabolic. Then $\left(R_{\leq}\right) \Rightarrow\left(R_{1}\right)$.

Proof. We need to prove (6.2) for any $u \in \mathcal{F} \cap C_{0}$ and all $x, y \in M$. If $u(x)=u(y)$, then (6.2) is trivially satisfied, so that we assume in the sequel that $u(x) \neq u(y)$.

Let $\left\{\Omega_{n}\right\}_{n=1}^{\infty}$ be an increasing sequence of precompact open sets that exhausts $M$. We can assume that all $\Omega_{n}$ contain $x$ and $y$. Since cap $\left(\Omega_{n}\right)=0$, there exists $\varphi_{n} \in \mathcal{F} \cap C_{0}$ such that $\left.\varphi_{n}\right|_{\Omega_{n}}=1$ and $\mathcal{E}\left(\varphi_{n}\right)<\frac{1}{n}$; in particular, $\mathcal{E}\left(\varphi_{n}\right) \rightarrow 0$ as $n \rightarrow \infty$.

For fixed $x, y$ as above, define the function $\widetilde{u}$ on $M$ by

$$
\widetilde{u}_{n}=\frac{u-u(y) \varphi_{n}}{u(x)-u(y)}
$$

It is obvious that $\widetilde{u}_{n} \in \mathcal{F} \cap C_{0}$ and $\widetilde{u}_{n}(x)=1, \widetilde{u}_{n}(y)=0$. Hence, by definition,

$$
\begin{aligned}
R(x, y)^{-1} & \leq \mathcal{E}\left(\widetilde{u}_{n}\right) \\
& =\frac{\mathcal{E}(u)-2 u(y) \mathcal{E}\left(u, \varphi_{n}\right)+u(y)^{2} \mathcal{E}\left(\varphi_{n}\right)}{(u(x)-u(y))^{2}} .
\end{aligned}
$$

Passing to the limit as $n \rightarrow \infty$, we obtain

$$
(u(x)-u(y))^{2} \leq R(x, y) \mathcal{E}(u) .
$$

Substituting here the estimate $\left(R_{\leq}\right)$we obtain (6.2). 
Define the condition $(R)$ as the conjunction of $\left(R_{\leq}\right)$and $\left(R_{\geq}\right)$. That is,

(R) : For all $x, y \in M$,

$$
R(x, y) \asymp d(x, y)^{\gamma} .
$$

We see from Propositions 6.5 and 6.6 that, under condition $(R)$,

$$
\text { parabolicity } \Leftrightarrow\left(R_{1}\right) \text {. }
$$

6.3. Condition $\left(R_{2}\right)$ with $\beta<2$.

Proposition 6.7. Let $(\mathcal{E}, \mathcal{F})$ be a regular Dirichlet form in $L^{2}$ with $\mathcal{E}^{(L)} \equiv 0$ and the jump density $J(x, y)$. Assume that $\left(V_{\leq}\right)$and $\left(J_{\leq}\right)$hold with some $0<\alpha<\beta<2$. Assume also that $\mathcal{F}$ contains all Lipschitz functions with compact supports. Then condition $\left(R_{2}\right)$ holds with $\gamma=\beta-\alpha$. Moreover, $(\mathcal{E}, \mathcal{F})$ is parabolic.

Remark 6.8. By hypothesis we have

$$
\mathcal{E}(\phi)=\mathcal{E}^{(J)}(\phi)=\int_{M \times M} \int_{M}(\phi(x)-\phi(y))^{2} J(x, y) d \mu(y) d \mu(x) .
$$

As we will see from the proof, the right hand side here is finite for any Lipschitz function $\phi$ with compact support, provided $\left(V_{\leq}\right)$and $\left(J_{\leq}\right)$hold. Therefore, the condition that $\mathcal{F}$ must contain all such functions is reasonable and not restrictive. In fact, one can define $\mathcal{F}$ as the closure of the space of Lipschitz functions with compact support.

The condition $\beta<2$ is closely related to the use of Lipschitz functions and cannot be dropped. It is not known if a similar statement is true for $\beta \geq 2$.

Proof. Set $B=B\left(x_{0}, r\right)$. Let $\phi$ be a Lipschitz function on $M$ with the Lipschitz constant $L$, such that $\phi \equiv 0$ in $B^{c}$. Then $\phi \in \mathcal{F}$. Since the function $|\phi(x)-\phi(y)|$ is symmetric in $x, y$ and vanishes if $(x, y) \in B^{c} \times B^{c}$, in particular, if $(x, y) \in$ $(2 B)^{c} \times(2 B)^{c}$, it follows that

$$
\begin{aligned}
\mathcal{E}(\phi) & =\mathcal{E}^{(J)}(\phi) \leq C \int_{M} \int_{M} \frac{(\phi(x)-\phi(y))^{2}}{d(x, y)^{\alpha+\beta}} d \mu(y) d \mu(x) \\
& \leq C\left(\int_{2 B} \int_{2 B}+2 \int_{2 B} \int_{(2 B)^{c}}\right) \frac{(\phi(x)-\phi(y))^{2}}{d(x, y)^{\alpha+\beta}} d \mu(y) d \mu(x) .
\end{aligned}
$$

Using $\left(V_{\leq}\right)$, the Lipschitz condition, and $2 B \subset B(x, 4 r)$, the first integral in (6.11) can be estimated by

$$
\begin{aligned}
L^{2} \int_{2 B}\left(\int_{B(x, 4 r)} \frac{d \mu(y)}{d(x, y)^{\alpha+\beta-2}}\right) d \mu(x) & \leq L^{2} \mu(2 B) \int_{0}^{4 r} s^{2-(\alpha+\beta)} \cdot s^{\alpha-1} d s \\
& \leq C L^{2} r^{\alpha-\beta+2},
\end{aligned}
$$

where we have also used $\beta<2$.

In the second integral in (6.11) we have $\phi(y)=0$. Since $\phi(x)=0$ outside $B$, the integration in $x$ can be reduced to $B$ instead of $2 B$. Hence, the second integral is estimated by

$$
\begin{aligned}
\int_{B} \phi(x)^{2}\left(\int_{B(x, r)^{c}} \frac{d \mu(y)}{d(x, y)^{\alpha+\beta}}\right) d \mu(x) & \leq C\|\phi\|_{\infty}^{2} \mu(B) \int_{r}^{\infty} s^{-(\alpha+\beta)} \cdot s^{\alpha-1} d s \\
& \leq C\|\phi\|_{\infty}^{2} r^{\alpha-\beta} .
\end{aligned}
$$


Hence, we obtain

$$
\mathcal{E}(\phi) \leq C L^{2} r^{\alpha-\beta+2}+C\|\phi\|_{\infty}^{2} r^{\alpha-\beta} .
$$

Specify the function $\phi$ by

$$
\phi(x)=\left(1-\frac{d\left(x_{0}, x\right)}{r}\right)_{+}
$$

and observe that it vanishes in $B^{c}$, has the Lipschitz constant $L=\frac{1}{r}$, and $\|\phi\|_{\infty}=$ $\varphi\left(x_{0}\right)=1$. It follows from (6.1) and 6.13) that

$$
R\left(x_{0}, B^{c}\right)^{-1} \leq \mathcal{E}(\phi) \leq C r^{\alpha-\beta},
$$

thus proving $\left(R_{2}\right)$.

Finally, for any compact subset $K$ of $M$, let $r_{n} \uparrow \infty$ as $n \rightarrow \infty$ so that $K \subset$ $B\left(x_{0}, r_{1} / 2\right)$. Set

$$
\phi_{n}(x)=\left(1-\frac{d\left(x_{0}, x\right)}{r_{n}}\right)_{+} .
$$

Then $\phi_{n} \geq \frac{1}{2}$ in $K$. Set $u_{n}=\left(2 \phi_{n}\right) \wedge 1$. We have

$$
\operatorname{cap}(K) \leq \mathcal{E}\left(u_{n}\right) \leq 4 \mathcal{E}\left(\phi_{n}\right) \leq \frac{C}{r_{n}^{\beta-\alpha}} \rightarrow 0
$$

as $n \rightarrow \infty$, showing that $\operatorname{cap}(K)=0$. Hence, $(\mathcal{E}, \mathcal{F})$ is parabolic.

6.4. Condition $\left(R_{2}\right)$ with general $\beta$. Fix some $0<\alpha<\beta$ and set $\gamma=\beta-\alpha$. In this section we obtain $\left(R_{2}\right)$ for an arbitrary value of $\beta$ at the expense of using more hypotheses.

Proposition 6.9. Let $(\mathcal{E}, \mathcal{F})$ be a regular Dirichlet form in $L^{2}$. Then

$$
\left(V_{\leq}\right)+\left(J_{\leq}\right)+\left(R_{\geq}\right)+\left(R_{1}\right) \Rightarrow\left(R_{2}\right) .
$$

Proof. We first claim that, for any $u_{1}, u_{2} \in \mathcal{F}$,

$$
\mathcal{E}\left(u_{1} \wedge u_{2}\right) \leq \mathcal{E}\left(u_{1}\right)+\mathcal{E}\left(u_{2}\right) .
$$

Indeed, set $v_{1}=u_{1}-u_{2}$ and $v_{2}=u_{1}+u_{2}$ so that

$$
u_{1} \wedge u_{2}=\frac{1}{2}\left(v_{2}-\left|v_{1}\right|\right)
$$

and

$$
\begin{aligned}
\mathcal{E}\left(u_{1} \wedge u_{2}\right) & =\frac{1}{4} \mathcal{E}\left(v_{2}-\left|v_{1}\right|\right)=\frac{1}{4}\left(\mathcal{E}\left(v_{2}\right)+\mathcal{E}\left(\left|v_{1}\right|\right)-2 \mathcal{E}\left(v_{2},\left|v_{1}\right|\right)\right) \\
& \leq \frac{1}{2}\left(\mathcal{E}\left(v_{2}\right)+\mathcal{E}\left(\left|v_{1}\right|\right)\right) \\
& \leq \frac{1}{2}\left(\mathcal{E}\left(v_{2}\right)+\mathcal{E}\left(v_{1}\right)\right) \\
& =\frac{1}{2}\left(\mathcal{E}\left(u_{1}+u_{2}\right)+\mathcal{E}\left(u_{1}-u_{2}\right)\right) \\
& =\mathcal{E}\left(u_{1}\right)+\mathcal{E}\left(u_{2}\right)
\end{aligned}
$$

which proves (6.14).

The following argument is motivated by [5, the proof of Lemma 2.4]. Fix a ball $B=B\left(x_{0}, r\right)$. For $x \in B \backslash \frac{1}{2} B$, let $\psi_{x} \in \mathcal{F}$ such that

$$
\psi_{x}\left(x_{0}\right)=1, \quad \psi_{x}(x)=0, \quad 0 \leq \psi_{x} \leq 1 \text { in } M,
$$


and

$$
\mathcal{E}\left(\psi_{x}\right)=R\left(x_{0}, x\right)^{-1}
$$

Fix some small enough $\eta>0$. It follows from $\left(R_{1}\right),\left(R_{\geq}\right)$that, for all $y \in B(x, \eta r)$,

$$
\begin{aligned}
\psi_{x}(y)^{2} & =\left|\psi_{x}(y)-\psi_{x}(x)\right|^{2} \leq C d(x, y)^{\gamma} \mathcal{E}\left(\psi_{x}\right) \\
& \leq C \frac{(\eta r)^{\gamma}}{R\left(x_{0}, x\right)} \leq c \eta^{\gamma} \leq \frac{1}{4}
\end{aligned}
$$

provided $\eta$ is small enough. Hence, we have $\psi_{x} \leq \frac{1}{2}$ in $B(x, \eta r)$ (cf. Figure 1). Since $\mu$ is $\alpha$-regular, there exists an integer $N$ independent of $x_{0}, r$ such that the

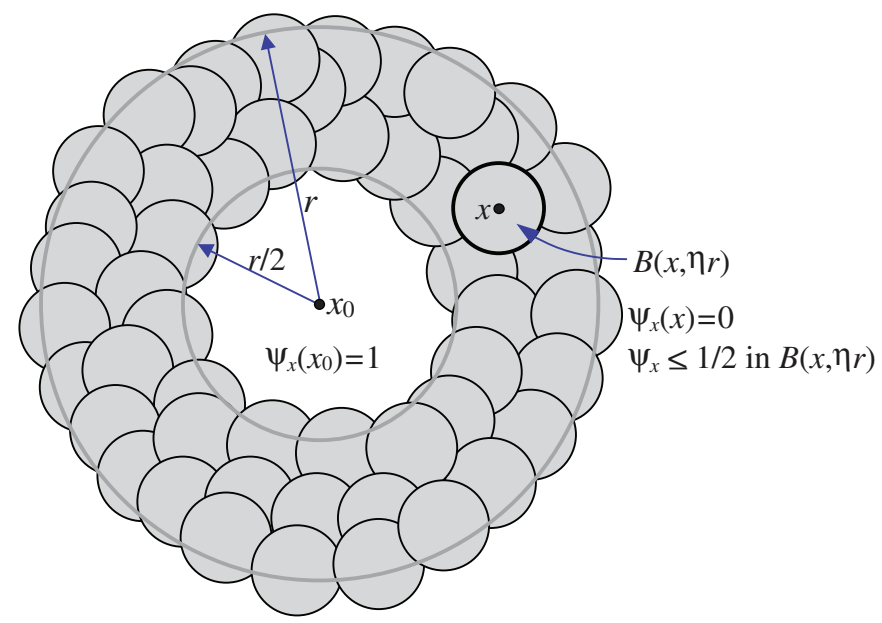

Figure 1. Function $\psi_{x}$ and covering for $B \backslash \frac{1}{2} B$

annulus $B \backslash \frac{1}{2} B$ can be covered by $N$ balls $\left\{B\left(x_{i}, \eta r\right)\right\}_{i=1}^{N}$. Define the function

$$
f=\psi_{x_{1}} \wedge \psi_{x_{2}} \wedge \cdots \wedge \psi_{x_{N}}
$$

and set

$$
g=2\left(f-\frac{1}{2}\right)_{+} .
$$

We have $f\left(x_{0}\right)=1$ and, by the above argument, $f \leq \frac{1}{2}$ in $B \backslash \frac{1}{2} B$. It follows that $g\left(x_{0}\right)=1, g \equiv 0$ in $B \backslash \frac{1}{2} B$, and $0 \leq g \leq 1$ in $M$. Using the Markov property of $(\mathcal{E}, \mathcal{F}),(6.14)$, and $\left(R_{\geq}\right)$, we have

$$
\mathcal{E}(g) \leq 4 \mathcal{E}(f) \leq 4 \sum_{i=1}^{N} \mathcal{E}\left(\psi_{x_{i}}\right)=4 \sum_{i=1}^{N} R\left(x_{0}, x_{i}\right)^{-1} \leq c r^{\alpha-\beta} .
$$

Consider the functions

$$
g_{1}=g \phi \text { and } g_{2}=g \mathbf{1}_{B^{c}},
$$

where $\phi$ is a cut-off function of the pair $\left(\frac{1}{2} B, B\right)$ (cf. Figure 2).

Observe that

$$
\operatorname{supp} g_{1} \subset \frac{1}{2} B, \quad g_{1}\left(x_{0}\right)=1, \quad \text { and } g=g_{1}+g_{2} .
$$




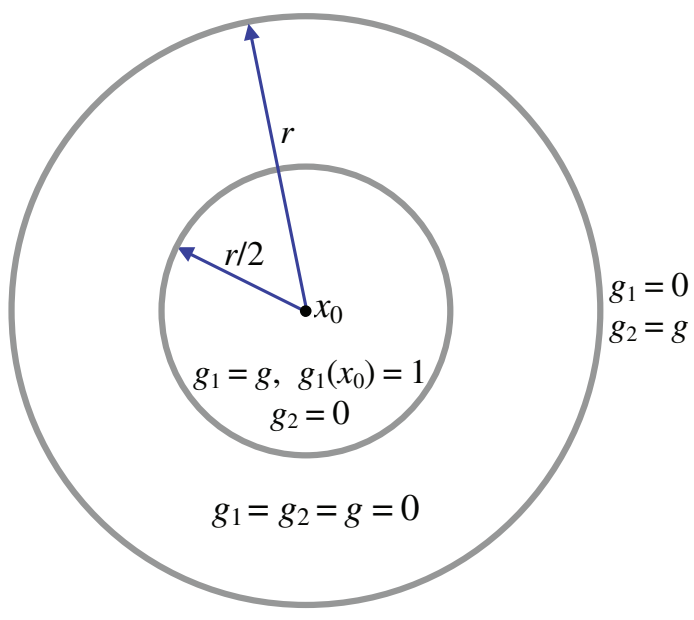

Figure 2. Functions $g_{1}$ and $g_{2}$

Also, $g_{1} \in \mathcal{F}$ by construction and $g_{2} \in \mathcal{F}$ because $g_{2}=g-g_{1}$. Since the supports of $g_{1}$ and $g_{2}$ are disjoint, we obtain

$$
\mathcal{E}^{(L)}(g)=\mathcal{E}^{(L)}\left(g_{1}+g_{2}\right)=\mathcal{E}^{(L)}\left(g_{1}\right)+\mathcal{E}^{(L)}\left(g_{2}\right) \geq \mathcal{E}^{(L)}\left(g_{1}\right) .
$$

On the other hand, using $\left(J_{\leq}\right)$and $\left(V_{\leq}\right)$and arguing as in (6.12), we obtain

$$
\begin{aligned}
\mathcal{E}^{(J)}\left(g_{1}, g_{2}\right) & =\int_{M \times M} \int_{M}\left(g_{1}(x)-g_{1}(y)\right)\left(g_{2}(x)-g_{2}(y)\right) J(x, y) d \mu(y) d \mu(x) \\
& =-2 \int_{\frac{1}{2} B} \int_{B^{c}} g_{1}(x) g_{2}(y) J(x, y) d \mu(y) d \mu(x) \\
& \geq-2 \int_{\frac{1}{2} B} \int_{B(x, r / 2)^{c}} g_{1}(x) g_{2}(y) J(x, y) d \mu(y) d \mu(x) \\
& \geq-c r^{\alpha-\beta}
\end{aligned}
$$

It follows that

$$
\begin{aligned}
\mathcal{E}^{(J)}(g) & =\mathcal{E}^{(J)}\left(g_{1}+g_{2}\right) \\
& =\mathcal{E}^{(J)}\left(g_{1}\right)+\mathcal{E}^{(J)}\left(g_{2}\right)+2 \mathcal{E}^{(J)}\left(g_{1}, g_{2}\right) \\
& \geq \mathcal{E}^{(J)}\left(g_{1}\right)-c r^{\alpha-\beta} .
\end{aligned}
$$

Combining (6.16), 6.17), and (6.15), we obtain

$$
\begin{aligned}
\mathcal{E}\left(g_{1}\right) & =\mathcal{E}^{(L)}\left(g_{1}\right)+\mathcal{E}^{(J)}\left(g_{1}\right) \\
& \leq \mathcal{E}^{(L)}(g)+\mathcal{E}^{(J)}(g)+c r^{\alpha-\beta} \\
& =\mathcal{E}(g)+c r^{\alpha-\beta} \\
& \leq C r^{\alpha-\beta} .
\end{aligned}
$$

Since $g_{1}$ is a test function for the resistance $R\left(x_{0}, B^{c}\right)$, we conclude that

$$
R\left(x_{0}, B^{c}\right)^{-1} \leq \mathcal{E}\left(g_{1}\right) \leq C r^{\alpha-\beta},
$$

which was to be proved. 
Corollary 6.10. Let $(\mathcal{E}, \mathcal{F})$ be a regular Dirichlet form in $L^{2}$. Then

$$
\text { parabolicity }+\left(V_{\leq}\right)+\left(J_{\leq}\right)+(R) \Rightarrow\left(R_{1}\right)+\left(R_{2}\right) .
$$

Proof. Indeed, by Proposition 6.6 we have

$$
\text { parabolicity }+\left(R_{\leq}\right) \Rightarrow\left(R_{1}\right),
$$

and the rest follows directly from Proposition 6.9.

6.5. The Green function. Assuming that $(M, d)$ is non-compact and condition $\left(R_{1}\right)$ is satisfied, we construct the Dirichlet Green function, vanishing outside a precompact open set $\Omega \subset M$. It turns out that it can be expressed in terms of effective resistance and harmonic function in $\Omega$.

By condition $\left(R_{1}\right)$, for any precompact open subset $\Omega$ of $M$, the space $\mathcal{F}(\Omega)$ is a Hilbert space under norm $\mathcal{E}(u)^{1 / 2}$ because, for $u \in \mathcal{F}(\Omega)$ and for $x \in \Omega, y \in M \backslash \Omega$, we have from $\left(R_{1}\right)$ that

$$
|u(x)|^{2}=|u(y)-u(x)|^{2} \leq C d(x, y)^{\gamma} \mathcal{E}(u),
$$

and thus the integration over $\Omega$ gives that $\|u\|_{2}^{2} \leq c(\Omega) \mathcal{E}(u)$. Therefore, by the Arzelà-Ascoli theorem, the embedding

$$
\mathcal{F}(\Omega) \hookrightarrow C(\bar{\Omega})
$$

is compact.

By the standard argument of PDE, it follows that, for any fixed precompact open subset $\Omega$ and any fixed $x \in \Omega$, the variational problem

$$
\inf \{\mathcal{E}(u): u \in \mathcal{F}(\Omega), u(x)=1\}
$$

possesses a solution, that is, there exists a function $\psi_{\Omega}(x, \cdot) \in \mathcal{F}(\Omega)$ satisfying that $\psi_{\Omega}(x, x)=1,0 \leq \psi_{\Omega}(x, \cdot) \leq 1$ on $M$, such that

$$
R\left(x, \Omega^{c}\right)^{-1}=\mathcal{E}\left(\psi_{\Omega}(x, \cdot)\right) .
$$

For any $x \in \Omega$, the function $\psi_{\Omega, x}(\cdot):=\psi_{\Omega}(x, \cdot)$ is harmonic in $M \backslash\left(\{x\} \cup \Omega^{c}\right)$ in the sense that

$$
\mathcal{E}\left(\psi_{\Omega, x}, \varphi\right)=0
$$

for any test function $\varphi \in \mathcal{F}(\Omega)$ with $\varphi(x)=0$, because the function $f:=\psi_{\Omega, x}+$ $\lambda \varphi \in \mathcal{F}(\Omega), f(x)=1$ for any real $\lambda$, and

$$
\begin{aligned}
\mathcal{E}\left(\psi_{\Omega, x}\right) & \leq \mathcal{E}(f)=\mathcal{E}\left(\psi_{\Omega, x}+\lambda \varphi\right) \\
& =\mathcal{E}\left(\psi_{\Omega, x}\right)+2 \lambda \mathcal{E}\left(\psi_{\Omega, x}, \varphi\right)+\lambda^{2} \mathcal{E}(\varphi),
\end{aligned}
$$

showing that $\mathcal{E}\left(\psi_{\Omega, x}, \varphi\right)=0$.

The effective resistance $R\left(x, \Omega^{c}\right)$ together with the harmonic function $\psi_{\Omega}(x, \cdot)$ gives rise to the Green function as follows:

$$
g_{\Omega}(x, y):=\left\{\begin{array}{c}
R\left(x, \Omega^{c}\right) \psi_{\Omega}(x, y), \text { if } x, y \in \Omega, \\
0, \text { otherwise. }
\end{array}\right.
$$

Such a way of defining a Green function was first addressed in [28, Section 4] if $\Omega$ (instead of a ball) is a finite subset of $M$, and then was extended to infinite subsets $\Omega$ in [29, Section 4]. See also that [23, Section 4] follows the same pattern as in [28] when $\Omega$ is infinite. 
Clearly, it follows from (6.21) that $g_{\Omega}(x, y) \geq 0$ for any $x, y \in M$, and $g_{\Omega}(x, \cdot) \in$ $\mathcal{F}(\Omega)$. Moreover, for any $\varphi \in \mathcal{F}(\Omega)$ and any fixed $x \in \Omega$,

$$
\mathcal{E}\left(g_{\Omega}(x, \cdot), \varphi\right)=\varphi(x),
$$

because, if $\varphi(x)=0$, then (6.22) follows directly from (6.20), and if $\varphi(x) \neq 0$, then (6.22) follows by replacing the function $\varphi(\cdot)$ in (6.20) by $\psi_{\Omega}(x, \cdot)-\varphi(\cdot) / \varphi(x)$ and then using (6.19) and (6.21). For any $x, y \in \Omega$, letting $\varphi(\cdot)=g_{\Omega}(y, \cdot)$ in (6.22), we obtain that

$$
\begin{aligned}
g_{\Omega}(y, x) & =\mathcal{E}\left(g_{\Omega}(x, \cdot), g_{\Omega}(y, \cdot)\right) \\
& =\mathcal{E}\left(g_{\Omega}(y, \cdot), g_{\Omega}(x, \cdot)\right)=g_{\Omega}(x, y),
\end{aligned}
$$

that is, the Green function $g_{\Omega}(x, y)$ is symmetric in $x, y \in \Omega$.

We estimate the Green function $g_{B}(x, y)$ for any ball $B$.

Proposition 6.11. Let $(\mathcal{E}, \mathcal{F})$ be a regular Dirichlet form in $L^{2}$ that satisfies $\left(R_{1}\right)$ and $\left(R_{2}\right)$. Assume that there exists a constant $N>1$ such that the annuli $B(x, N r) \backslash B(x, r)$ are non-empty for all $x \in M$ and $r>0$. Then there exist constants $C>0, \eta \in(0,1)$ such that, for all balls $B=B\left(x_{0}, r\right)$,

$$
\begin{aligned}
g_{B}(x, y) & \leq C r^{\gamma} \text { for all } x, y \in B, \\
g_{B}\left(x_{0}, y\right) & \geq C^{-1} r^{\gamma} \text { for all } y \in B\left(x_{0}, \eta r\right) .
\end{aligned}
$$

Proof. Set $\Omega=B=B\left(x_{0}, r\right)$ and let $\psi_{B}(x, \cdot)$ be as in 6.19). Choosing a point $z \in 2 B \backslash B$ and using $\left(R_{1}\right)$, we obtain, for any $u \in \mathcal{F} \cap C_{0}$ such that $u\left(x_{0}\right)=1$ and $\left.u\right|_{B^{c}}=0$, that

$$
1=\left(u\left(x_{0}\right)-u(z)\right)^{2} \leq C d(x, z)^{\gamma} \mathcal{E}(u) \leq C r^{\gamma} \mathcal{E}(u),
$$

whence

$$
R\left(x_{0}, B^{c}\right)^{-1}=\inf \mathcal{E}(u) \geq C^{-1} r^{-\gamma}
$$

and $R\left(x_{0}, B^{c}\right) \leq C r^{\gamma}$. Applying the latter inequality to the ball $B(x, 2 r)$ instead of $B\left(x_{0}, r\right)$, we obtain, for any $x \in B$,

$$
R\left(x, B^{c}\right) \leq R\left(x, B(x, 2 r)^{c}\right) \leq C r^{\gamma} .
$$

Substituting this estimate into (6.21) and using $0 \leq \psi_{B}(x, \cdot) \leq 1$ we obtain (6.24).

To show (6.25) observe that by (6.19) and $\left(R_{2}\right)$

$$
\mathcal{E}\left(\psi_{B}\left(x_{0}, \cdot\right)\right)=R\left(x_{0}, B^{c}\right)^{-1} \leq c r^{-\gamma} .
$$

Then, using $\left(R_{1}\right)$, we obtain, for any $y \in B\left(x_{0}, \eta r\right)$,

$$
\begin{aligned}
\left(1-\psi_{B}\left(x_{0}, y\right)\right)^{2} & =\left(\psi_{B}\left(x_{0}, x_{0}\right)-\psi_{B}\left(x_{0}, y\right)\right)^{2} \\
& \leq C d\left(x_{0}, y\right)^{\gamma} \mathcal{E}\left(\psi_{B}\left(x_{0}, \cdot\right)\right) \\
& \leq C\left(\frac{d\left(x_{0}, y\right)}{r}\right)^{\gamma} \\
& \leq C \eta^{\gamma} .
\end{aligned}
$$

If $\eta$ is small enough, then $C \eta^{\gamma}<\frac{1}{4}$, whence it follows that

$$
\psi_{B}\left(x_{0}, y\right) \geq \frac{1}{2} \text { in } B\left(x_{0}, \eta r\right) .
$$


Using again (6.21) and $\left(R_{2}\right)$, we obtain, for all $y \in B\left(x_{0}, \eta r\right)$,

$$
g_{B}\left(x_{0}, y\right)=R\left(x_{0}, B^{c}\right) \psi_{B}\left(x_{0}, y\right) \geq \frac{1}{2} c r^{\gamma},
$$

which proves (6.25).

Let $\mathcal{L}_{B}$ be the generator of $(\mathcal{E}, \mathcal{F}(B))$. Given $f \in L^{2}(B)$, consider the function

$$
u(x)=\int_{B} g_{B}(x, y) f(y) d \mu(y),
$$

where $g_{B}(x, y)$ is the Green function defined by 6.21). Then $u \in \mathcal{F}(B)$ and $u$ is a weak solution to the Poisson-type equation

$$
-\mathcal{L}_{B} u=f \text { in } B
$$

that is, for any $\varphi \in \mathcal{F}(B)$,

$$
\mathcal{E}(u, \varphi)=\int_{B} f(x) \varphi(x) d \mu(x) .
$$

Indeed, by (6.22) and the dominated convergence theorem, we obtain that, for any $\varphi \in \mathcal{F}(B)$,

$$
\begin{aligned}
\mathcal{E}(u, \varphi) & =\lim _{t \rightarrow 0} \frac{1}{t}\left(u-P_{t} u, \varphi\right) \\
& =\lim _{t \rightarrow 0} \frac{1}{t} \int_{B}\left(g_{B}(\cdot, y)-P_{t}\left(g_{B}(\cdot, y)\right), \varphi\right) f(y) d \mu(y) \\
& =\int_{B} \mathcal{E}\left(g_{B}(\cdot, y), \varphi\right) f(y) d \mu(y) \\
& =\int_{B} \varphi(y) f(y) d \mu(y),
\end{aligned}
$$

that proves (6.28).

Proposition 6.12. Assume that $\mu$ is $\alpha$-regular and $(\mathcal{E}, \mathcal{F})$ satisfies the conditions $\left(R_{1}\right)$ and $\left(R_{2}\right)$. Let $f$ be any cut-off function for the pair $\left(\frac{1}{2} B, B\right)$, where $B=$ $B\left(x_{0}, r\right)$ is an arbitrary ball. Then the solution $u$ defined by (6.26) satisfies the following estimates:

$$
u(x) \leq C r^{\gamma+\alpha} \text { in } B\left(x_{0}, r\right)
$$

and

$$
u(x) \geq c r^{\gamma+\alpha} \text { in } B\left(x_{0}, \eta r\right),
$$

where $C, c>0$ and $\eta \in(0,1 / 2)$ are constants that depend only on the constants in the hypotheses (and do not depend on the ball $B$ ).

Proof. Observe that the $\alpha$-regularity of $\mu$ implies that the annuli $B(x, N r) \backslash B(x, r)$ are non-empty provided $N$ is a large enough constant. Hence, Proposition 6.11 holds in the present setting. By reducing the constant $\eta$ from the statement of this proposition, we can assume that $\eta<\frac{1}{2}$.

It follows from (6.24) and $(\underline{6.26)}$ that, using the fact that $0 \leq f \leq 1$ in $M$,

$$
\begin{aligned}
u(x) & =\int_{B} g_{B}(x, y) f(y) d \mu(y) \leq \int_{B} g_{B}(x, y) d \mu(y) \\
& \leq C r^{\gamma} \mu(B) \leq C r^{\gamma+\alpha} .
\end{aligned}
$$


Similarly, observing that $f=1$ in $\eta B$ and using (6.25), we obtain

$$
\begin{aligned}
u\left(x_{0}\right) & =\int_{B} g_{B}\left(x_{0}, y\right) f(y) d \mu(y) \\
& \geq \int_{B\left(x_{0}, \eta r\right)} g_{B}\left(x_{0}, y\right) d \mu(y) \\
& \geq c r^{\gamma} \mu\left(B\left(x_{0}, \eta r\right)\right) \geq c_{1} r^{\gamma+\alpha} .
\end{aligned}
$$

Taking $\varphi=u$ in (6.28) and using (6.31) we obtain

$$
\mathcal{E}(u)=\int_{B} f(x) u(x) d \mu(x) \leq C r^{\gamma+\alpha} \mu(B) \leq c r^{\gamma+2 \alpha} .
$$

By Lemma 6.1, function $u$ satisfies (6.2), so that for any $x \in B\left(x_{0}, \eta r\right)$,

$$
\begin{aligned}
\left|u(x)-u\left(x_{0}\right)\right|^{2} & \leq c d\left(x, x_{0}\right)^{\gamma} \mathcal{E}(u) \\
& \leq C r^{\gamma+2 \alpha} d\left(x, x_{0}\right)^{\gamma} \\
& \leq C \eta^{\gamma} r^{2(\gamma+\alpha)} .
\end{aligned}
$$

Choosing $\eta$ small enough and comparing with (6.32), we obtain that

$$
\left|u(x)-u\left(x_{0}\right)\right|<\frac{1}{2} u\left(x_{0}\right),
$$

whence $u(x) \geq \frac{1}{2} u\left(x_{0}\right) \geq c r^{\gamma+\alpha}$ follows.

6.6. Upper bounds of the heat kernel under $\left(R_{1}\right)$ and $\left(R_{2}\right)$. We now use the estimates in (6.29)-(6.30) to obtain the survival estimate $(S)$ and $(D U E)$.

Theorem 6.13. Let $(\mathcal{E}, \mathcal{F})$ be a regular Dirichlet form in $L^{2}(M, \mu)$. Assume that $\mu$ is $\alpha$-regular and conditions $\left(R_{1}\right),\left(R_{2}\right)$ are satisfied with some $\gamma>0$. Then the survival estimate $(S)$ and $(D U E)$ hold with $\beta=\alpha+\gamma$. Consequently, we have

$$
(U E) \Leftrightarrow\left(J_{\leq}\right) .
$$

Proof. Let us first prove $(S)$. Fix a ball $B=B\left(x_{0}, r\right)$. We claim that for all $t>0$ and $\mu$-almost all $x \in B$,

$$
P_{t}^{B} 1_{B}(x) \geq \frac{u(x)-t}{\|u\|_{\infty}},
$$

where $u$ is given by (6.26) with $f$ being a cut-off function of the pair $\left(\frac{1}{2} B, B\right)$. Indeed, fix a cut-off function $\phi$ of the pair $(B, M)$ and consider the function

$$
w(t, x)=u(x)-t \phi(x)-\|u\|_{\infty} P_{t}^{B} 1_{B}(x) .
$$

Clearly, 6.34) is equivalent to $w \leq 0$. Let us first show that $w(t, x)$ is a weak subsolution of the heat equation in $(0, \infty) \times B$. We use the following facts:

(1) $P_{t}^{B} 1_{B}$ is a weak solution of the heat equation in $(0, \infty) \times B$; in particular,

$$
\left(\frac{\partial}{\partial t} P_{t}^{B} 1_{B}, \varphi\right)+\mathcal{E}\left(P_{t}^{B} 1_{B}, \varphi\right)=0
$$

for any $\varphi \in \mathcal{F}(B)$.

(2) $\mathcal{E}(\phi, \varphi) \geq 0$ for any $0 \leq \varphi \in \mathcal{F}(B)$ (see (4.9)). 
Using these as well as (6.28) we obtain for any $0 \leq \varphi \in \mathcal{F}(B)$ and $t>0$,

$$
\begin{aligned}
\left(\frac{\partial w}{\partial t}, \varphi\right)+\mathcal{E}(w, \varphi)= & \left(-\phi-\|u\|_{\infty} \frac{\partial}{\partial t} P_{t}^{B} 1_{B}, \varphi\right) \\
& +\mathcal{E}\left(u-t \phi-\|u\|_{\infty} P_{t}^{B} 1_{B}, \varphi\right) \\
= & -(\phi, \varphi)+\mathcal{E}(u, \varphi)-t \mathcal{E}(\phi, \varphi) \\
\leq & (f-\phi, \varphi) \leq 0,
\end{aligned}
$$

which proves that $w(t, x)$ is a weak subsolution of the heat equation in $(0, \infty) \times B$.

On the other hand, we have as $t \rightarrow 0+$

$$
w(t, \cdot) \stackrel{L^{2}(B)}{\rightarrow} u-\|u\|_{\infty} 1_{B} \leq 0,
$$

and hence $w_{+}$satisfies the initial condition

$$
w_{+}(t, \cdot) \stackrel{L^{2}(B)}{\rightarrow} 0
$$

Therefore, by the parabolic maximum principle of Proposition 7.1 we conclude that $w \leq 0$ for all $t>0$ and $\mu$-almost all $x \in B$, thus proving (6.34).

Using (6.34) and the estimates (6.29)- 6.30) of Proposition 6.12, we obtain that, for $\mu$-almost all $x \in B\left(x_{0}, \eta r\right)$,

$$
\begin{aligned}
P_{t}^{B} 1_{B}(x) & \geq \frac{u(x)-t}{\|u\|_{\infty}} \\
& \geq c-c_{1} t r^{-\beta} \\
& \geq \frac{c}{2},
\end{aligned}
$$

provided $t r^{-\beta}$ is small enough. This proves $(S)$ although with constant $\eta$ in (2.9) instead of $\frac{1}{4}$. Using the ball covering argument as in the proof of Theorem 3.1 one can replace a small value of $\eta$ by any value $<1$, in particular, by $\frac{1}{4}$.

Next we show that condition $\left(R_{1}\right)$ implies $(D U E)$. It suffices to show that condition $\left(R_{1}\right)$ implies the Faber-Krahn inequality

$$
\lambda_{\min }(\Omega):=\inf _{f \in \mathcal{F} \cap C_{0}(\Omega)} \frac{\mathcal{E}(f)}{\|f\|_{2}^{2}} \geq c \mu(\Omega)^{-\beta / \alpha}
$$

for all non-empty bounded 2 open subsets $\Omega$ of $M$, which in turn implies ( $D U E$ ) (see for example [16, Lemmas 5.4 and 5.5]). Motivated by the argument in [22, Lemma 4.2], consider a function $f \in \mathcal{F} \cap C_{0}(\Omega)$ normalized so that $\sup |f|=1$, and let $x \in \Omega$ be a point such that $|f(x)|=1$. Let $r$ be the largest radius such that $B(x, r) \subset \Omega$. Then the ball $B(x, 2 r)$ is not covered by $\Omega$ so that there exists a point $y \in B(x, 2 r) \backslash \Omega$ (note that $M$ is unbounded by condition $(V)$ ). In particular, $y \notin \operatorname{supp} f$ (see Figure 3 ).

Noting that $\mathcal{E}^{(J)}(f) \leq \mathcal{E}(f)$ and by the $\alpha$-regularity of $\mu$,

$$
r \leq C[\mu(B(x, r))]^{1 / \alpha} \leq C[\mu(\Omega)]^{1 / \alpha},
$$

\footnotetext{
${ }^{2}$ If 6.35 is satisfied for bounded $\Omega$, then 6.35 is also satisfied for all open $\Omega$ by the exhaustion argument.
} 


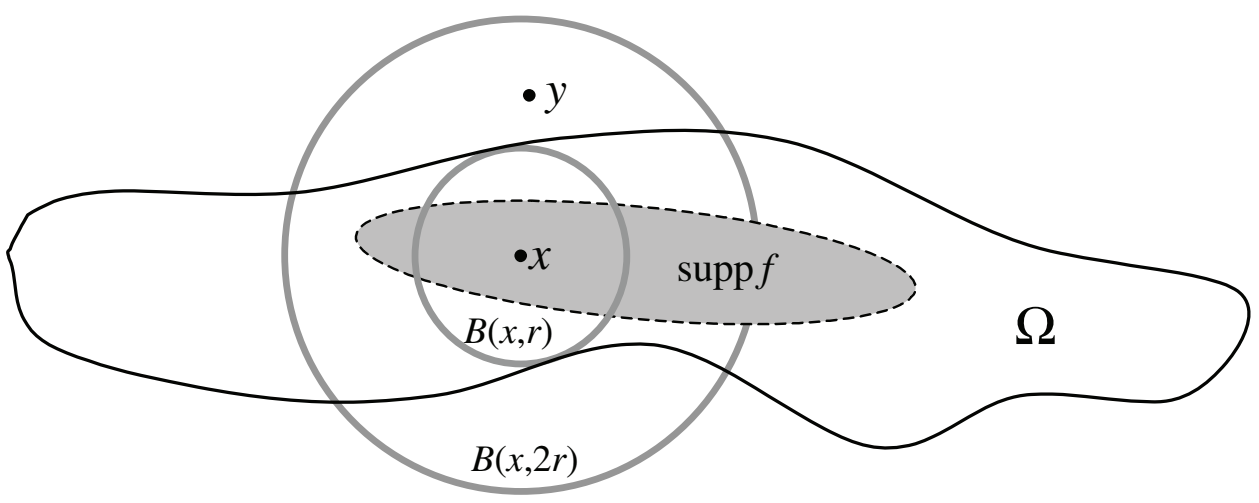

Figure 3

we obtain from $\left(R_{1}\right)$ that

$$
\begin{aligned}
1 & =|f(y)-f(x)|^{2} \\
& \leq C d(y, x)^{\beta-\alpha} \mathcal{E}^{(J)}(f) \\
& \leq C(2 r)^{\beta-\alpha} \mathcal{E}(f) \leq C 2^{\beta-\alpha}[\mu(\Omega)]^{\beta / \alpha-1} \mathcal{E}(f) .
\end{aligned}
$$

Since $\|f\|_{2}^{2} \leq \mu(\Omega)$, it follows that

$$
\frac{\mathcal{E}(f)}{\|f\|_{2}^{2}} \geq c[\mu(\Omega)]^{-\beta / \alpha},
$$

for some $c>0$, thus proving the Faber-Krahn inequality.

Finally, observe that $(\mathcal{E}, \mathcal{F})$ is parabolic by Proposition 6.5, and hence is conservative by Lemma 6.4. Therefore, the equivalence (6.33) follows directly from Theorem 2.3 .

Corollary 6.14. Let $(\mathcal{E}, \mathcal{F})$ be a regular Dirichlet form in $L^{2}$ with a jump density $J(x, y)$ and let $0<\alpha<\beta$. Then

$$
(V)+(J)+\left(R_{2}\right) \Rightarrow(U E) .
$$

Proof. Note that $(V)+(J) \Rightarrow\left(R_{1}\right)$ by Proposition 6.2. Hence, the conclusion (6.36) follows from Theorem 6.13 .

Remark 6.15. Besides the hypotheses of Corollary 6.14 if in addition $\mathcal{E}^{(L)} \equiv 0$ and $\beta<2$, and if $\mathcal{F}$ contains all Lipschitz functions with compact supports, then condition $\left(R_{2}\right)$ also holds by Proposition 6.7. Therefore, it follows from (6.36) that

$$
(V)+(J) \Rightarrow(U E) \text {. }
$$

Note that conclusion (6.37) was addressed in [6, 9].

Example 6.16. Let $M$ be the Sierpinski gasket in $\mathbb{R}^{n}$ and let

$$
\begin{aligned}
\mathcal{E}(f) & =\int_{M} \int_{M} \frac{(f(x)-f(y))^{2}}{|x-y|^{\alpha+\beta}} d \mu(y) d \mu(x), \\
\mathcal{F} & =\left\{f \in L^{2}(M, \mu): \mathcal{E}(f)<\infty\right\},
\end{aligned}
$$


where $\alpha=\frac{\log (n+1)}{\log 2}$ is the Hausdorff dimension of $M, \mu$ is the $\alpha$-dimensional Hausdorff measure on $M$ (so that condition $(V)$ holds), and

$$
\alpha<\beta<\beta_{*}:=\frac{\log (n+3)}{\log 2}
$$

(possibly $\beta \geq 2$ ). Then $(\mathcal{E}, \mathcal{F})$ is a regular Dirichlet form in $L^{2}$ and condition $(J)$ is satisfied. Note that condition $\left(R_{1}\right)$ is true where $\gamma=\beta-\alpha$ (see [17, Theorem 4.11 (iii), p. 2085]). In order to obtain $(U E)$, by Corollary 6.14 and Proposition 6.9, it suffices to verify condition $\left(R_{\geq}\right)$with $\gamma=\beta-\alpha$.

Indeed, let $\left(\mathcal{E}_{0}, \mathcal{F}_{0}\right)$ be the standard local, conservative, self-similar Dirichlet form on the Sierpinski gasket, and let $R_{0}$ be the associated effective resistance with respect to $\left(\mathcal{E}_{0}, \mathcal{F}_{0}\right)$. It is known (cf. [25]) that

$$
\begin{aligned}
R_{0}(x, y) & \asymp|x-y|^{\beta_{*}-\alpha}, \\
\mathcal{E}_{0}(f) & \asymp \sup _{r>0} r^{-\left(\alpha+\beta_{*}\right)} \int_{M} \int_{B(x, r)}(f(x)-f(y))^{2} d \mu(y) d \mu(x) .
\end{aligned}
$$

For any $r>0$, we have that

$$
\begin{aligned}
& \mathcal{E}_{1}(f):=\int_{M} \int_{B(x, r)} \frac{(f(x)-f(y))^{2}}{|x-y|^{\alpha+\beta}} d \mu(y) d \mu(x) \\
&=\sum_{k=0}^{\infty} \int_{M} \int_{B\left(x, 2^{-k} r\right) \backslash B\left(x, 2^{-k-1} r\right)} \frac{(f(x)-f(y))^{2}}{|x-y|^{\alpha+\beta}} d \mu(y) d \mu(x) \\
& \leq \sum_{k=0}^{\infty}\left(2^{-k-1} r\right)^{-\alpha-\beta} \int_{M} \int_{B\left(x, 2^{-k} r\right)}(f(x)-f(y))^{2} d \mu(y) d \mu(x) \\
& \leq \sum_{k=0}^{\infty}\left(2^{-k-1} r\right)^{-\alpha-\beta} \cdot\left[\left(2^{-k} r\right)^{\alpha+\beta_{*}} \mathcal{E}_{0}(f)\right] \quad(\text { using (6.39) }) \\
& \leq c r^{\beta_{*}-\beta} \mathcal{E}_{0}(f) .
\end{aligned}
$$

On the other hand, we have that, using condition $(V)$,

$$
\begin{aligned}
\mathcal{E}_{2}(f) & :=\int_{M} \int_{B(x, r)^{c}} \frac{(f(x)-f(y))^{2}}{|x-y|^{\alpha+\beta}} d \mu(y) d \mu(x) \\
& \leq \int_{M} \int_{B(x, r)^{c}} \frac{2\left(f(x)^{2}+f(y)^{2}\right)}{|x-y|^{\alpha+\beta}} d \mu(y) d \mu(x) \\
& \leq C r^{-\beta}\|f\|_{2}^{2} .
\end{aligned}
$$

It follows that, for all $r>0$,

$$
\begin{aligned}
\mathcal{E}(f) & =\mathcal{E}_{1}(f)+\mathcal{E}_{2}(f) \\
& \leq C\left[r^{\beta_{*}-\beta} \mathcal{E}_{0}(f)+r^{-\beta}\|f\|_{2}^{2}\right] .
\end{aligned}
$$

Minimizing the right-hand side of (6.40), we conclude that

$$
\mathcal{E}(f) \leq c\left(\|f\|_{2}^{2}\right)^{\left(\beta_{*}-\beta\right) / \beta_{*}} \cdot \mathcal{E}_{0}(f)^{\beta / \beta_{*}} .
$$

Now fix two points $x_{0}, y_{0} \in M$, and let $r:=\frac{\left|x_{0}-y_{0}\right|}{2}$. By (6.38), we can choose the test function $f$ such that $f\left(x_{0}\right)=1,\left.f\right|_{B\left(x_{0}, r\right)^{c}}=0$, and

$$
\mathcal{E}_{0}(f) \asymp R_{0}\left(x_{0}, y_{0}\right)^{-1} \asymp r^{-\left(\beta_{*}-\alpha\right)} .
$$


Thus, using the Faber-Krahn inequality $\lambda_{\min }\left(B\left(x_{0}, r\right)\right) \geq c r^{-\beta_{*}}$, we have that

$$
\|f\|_{2}^{2} \leq \frac{\mathcal{E}_{0}(f)}{\lambda_{\min }\left(B\left(x_{0}, r\right)\right)} \leq c r^{\beta_{*}} \mathcal{E}_{0}(f) \leq C r^{\alpha} .
$$

Combining this with (6.41), we obtain that

$$
\begin{aligned}
\mathcal{E}(f) & \leq c\left(C r^{\alpha}\right)^{\left(\beta_{*}-\beta\right) / \beta_{*}} \cdot \mathcal{E}_{0}(f)^{\beta / \beta_{*}} \\
& \leq C\left(C r^{\alpha}\right)^{\left(\beta_{*}-\beta\right) / \beta_{*}} \cdot r^{-\left(\beta_{*}-\alpha\right) \beta / \beta_{*}}=C^{\prime} r^{\alpha-\beta},
\end{aligned}
$$

and hence

$$
\begin{aligned}
R\left(x_{0}, y_{0}\right)^{-1} & \leq \mathcal{E}(f) \leq C^{\prime} r^{\alpha-\beta} \\
& =C^{\prime}\left(\frac{\left|x_{0}-y_{0}\right|}{2}\right)^{\alpha-\beta}
\end{aligned}
$$

that is, $R\left(x_{0}, y_{0}\right) \geq c\left|x_{0}-y_{0}\right|^{\beta-\alpha}$. This proves that condition $\left(R_{\geq}\right)$holds.

6.7. Two-sided estimates of the heat kernel. Let us introduce the following condition:

(NLE) : (The near diagonal lower estimate) There exist $\delta \in(0,1)$ and $c>0$ such that, for all $t>0$ and almost all $x, y \in M$ such that $d(x, y) \leq \delta t^{1 / \beta}$,

$$
p_{t}(x, y) \geq c t^{-\alpha / \beta} \text {. }
$$

Theorem 6.17. Let $(\mathcal{E}, \mathcal{F})$ be a regular Dirichlet form in $L^{2}(M, \mu)$. Fix parameters $0<\alpha<\beta$ and set $\gamma=\beta-\alpha$. Then the following equivalences are true:

$$
\begin{aligned}
\text { parabolicity }+(V)+\left(J_{\leq}\right)+(R) & \Leftrightarrow(U E)+(N L E) \\
& \Leftrightarrow(U E)+(N L E)+\text { continuity, }
\end{aligned}
$$

where "continuity" means that for any $t>0$ the heat kernel $p_{t}(x, y)$ is continuous in $(x, y) \in M \times M$.

Proof. We first show the implication

$$
\text { parabolicity }+(V)+\left(J_{\leq}\right)+(R) \Rightarrow(U E)+(N L E)+\text { continuity. }
$$

By Corollary 6.10 both conditions $\left(R_{1}\right)$ and $\left(R_{2}\right)$ are satisfied. Hence, by Theorem 6.13, we obtain $(U E)$.

Let us show that $p_{t}(x, y)$ as a function of $x, y$ admits a continuous version. By $\left(R_{1}\right)$ and Lemma 6.1, all functions from $\mathcal{F}$ are Hölder continuous and, moreover, satisfy (6.2). By $(U E)$ we have, for any $f \in L^{2}$,

$$
\left\|P_{t} f\right\|_{L^{\infty}} \leq C t^{-\alpha / \beta}\|f\|_{2} .
$$

Since $P_{t} f \in \mathcal{F}$, it admits a continuous version that will also be denoted by $P_{t} f$. Then we can write, for all $t>0$ and $x \in M$,

$$
\left|P_{t} f(x)\right| \leq C t^{-\alpha / \beta}\|f\|_{2} .
$$

By the Riesz representation theorem, there is a function $p_{t}(x, \cdot) \in L^{2}$ such that

$$
P_{t} f(x)=\left(p_{t}(x, \cdot), f\right) .
$$


The function $p_{t}(x, y)$ as a function of $x, y$ is symmetric in the following sense: $p_{t}(x, y)=p_{t}(y, x)$ for almost all $x, y \in M$. Using the semigroup identity $P_{t+s}=$ $P_{t} P_{s}$ and the symmetry of the heat kernel, we obtain

$$
\begin{aligned}
\left(p_{t+s}(x, \cdot), f\right) & =\int_{M} p_{t}(x, z)\left(\int_{M} p_{s}(z, y) f(y) d \mu(y)\right) d \mu(z) \\
& =\int_{M}\left(\int_{M} p_{t}(x, z) p_{s}(y, z) d \mu(z)\right) f(y) d \mu(y)
\end{aligned}
$$

whence

$$
p_{t+s}(x, y)=\int_{M} p_{t}(x, z) p_{s}(y, z) d \mu(z)=\left(p_{t}(x, \cdot), p_{s}(y, \cdot)\right)
$$

for almost all $y$. The right-hand side is well defined for all $x, y \in M$ and is continuous separately in $x$ and in $y$ because it has the form (6.42). Therefore, the left-hand side, that is, $p_{t+s}(x, y)$, has a pointwise version that is continuous in $x$ and $y$ separately. Switching to this version we obtain, in particular, that the identity (6.43) is true pointwise.

On the other hand, using the spectral resolution $\left\{E_{\lambda}\right\}$ of the generator $\mathcal{L}$ and the elementary inequality $\lambda e^{-2 \lambda t} \leq \frac{1}{2 e t}$ that is true for all $\lambda \geq 0$ and $t>0$, we obtain

$$
\mathcal{E}\left(P_{t} f\right)=\int_{0}^{\infty} \lambda e^{-2 \lambda t} d\left(E_{\lambda} f, f\right) \leq \frac{1}{2 e t}\|f\|_{2}^{2} \text { for } f \in L^{2}
$$

Applying this inequality with $f=p_{t}(x, \cdot)$ and using $(U E)$ and (6.43) yields

$$
\begin{aligned}
\mathcal{E}\left(p_{2 t}(x, \cdot)\right) & \leq \frac{1}{2 e t}\left\|p_{t}(x, \cdot)\right\|_{2}^{2} \\
& =\frac{1}{2 e t} p_{2 t}(x, x) \leq C t^{-1-\alpha / \beta},
\end{aligned}
$$

for all $t>0$ and $x \in M$.

Since by (6.43) $p_{t+s}(x, \cdot) \in \mathcal{F}$, we obtain by (6.2) and (6.44) that

$$
\left|p_{t}\left(x, y_{1}\right)-p_{t}\left(x, y_{2}\right)\right|^{2} \leq C d\left(y_{1}, y_{2}\right)^{\gamma} \mathcal{E}\left(p_{t}(x, \cdot)\right) \leq C t^{-1-\alpha / \beta} d\left(y_{1}, y_{2}\right)^{\gamma}
$$

so that the function $p_{t}(x, y)$ is continuous in $y$ uniformly in $x$. Hence, $p_{t}(x, y)$ is continuous jointly in $x, y$.

To verify $(N L E)$, we use a standard argument. It follows from $(U E)$ and the $\alpha$-regularity of $\mu$ that, for all $x \in M$ and $t, r>0$,

$$
\int_{B(x, r)^{c}} p_{t}(x, y) d \mu(y) \leq c \int_{r t^{-1 / \beta} / 2}^{\infty} s^{\alpha-1}(1+s)^{-(\alpha+\beta)} d s \leq \frac{1}{2}
$$

provided $r t^{-1 / \beta} \geq C$ for some large $C$. Choosing $r=C t^{1 / \beta}$, we obtain

$$
\int_{B(x, r)} p_{t}(x, y) d \mu(y)=1-\int_{B(x, r)^{c}} p_{t}(x, y) d \mu(y) \geq \frac{1}{2} .
$$


By the semigroup property, the symmetry of $p_{t}$ and the Cauchy-Schwarz inequality, we have

$$
\begin{aligned}
p_{2 t}(x, x) & =\int_{M} p_{t}(x, y)^{2} d \mu(y) \\
& \geq \frac{1}{V(x, r)}\left[\int_{B(x, r)} p_{t}(x, y) d \mu(y)\right]^{2} \\
& \geq c r^{-\alpha}=c_{1} t^{-\alpha / \beta} .
\end{aligned}
$$

Combining (6.44) with $\left(R_{1}\right)$ we obtain, for all $y \in B\left(x, \delta t^{1 / \beta}\right)$,

$$
\begin{aligned}
\left|p_{2 t}(x, x)-p_{2 t}(x, y)\right|^{2} & \leq C d(x, y)^{\beta-\alpha} \mathcal{E}\left(p_{2 t}(x, \cdot)\right) \\
& \leq C d(x, y)^{\beta-\alpha} t^{-1-\alpha / \beta} \\
& \leq C \delta^{\beta-\alpha} t^{-2 \alpha / \beta}
\end{aligned}
$$

If $\delta$ is small enough, then $C \delta^{\beta-\alpha}<\frac{1}{4} c_{1}^{2}$ where $c_{1}$ is the constant in 6.45. It follows that

$$
p_{2 t}(x, y) \geq p_{2 t}(x, x)-\frac{c_{1} t^{-\alpha / \beta}}{2} \geq \frac{c_{1} t^{-\alpha / \beta}}{2},
$$

thus proving $(N L E)$.

Next we show the opposite implication,

$$
(U E)+(N L E) \Rightarrow \text { parabolicity }+(V)+\left(J_{\leq}\right)+(R) .
$$

Note that $(\mathcal{E}, \mathcal{F})$ is conservative by Lemma 6.4 By [17, Theorem 3.2], $(U E)+$ $(N L E) \Rightarrow(V)$. By Theorem 2.3 , we have $(U E) \Rightarrow\left(J_{\leq}\right)$. To show $\left(R_{1}\right)$, we use the following inequality:

$$
\mathcal{E}(u) \geq \frac{1}{2 t} \int_{M} \int_{M}(u(x)-u(y))^{2} p_{t}(x, y) d \mu(y) d \mu(x),
$$

that is true for any $u \in \mathcal{F}$ and for any $t>0$ (see [17]). Fix some $r>0$ and choose $t$ from the identity $r=\delta t^{1 / \beta}$. It follows from $(N L E)$ that, for any $u \in \mathcal{F}$,

$$
\begin{aligned}
\mathcal{E}(u) & \geq \frac{1}{2 t} \int_{M} \int_{B(x, r)}(u(x)-u(y))^{2} p_{t}(x, y) d \mu(y) d \mu(x) \\
& \geq \frac{c}{2 t^{1+\alpha / \beta}} \int_{M} \int_{B(x, r)}(u(x)-u(y))^{2} d \mu(y) d \mu(x) \\
& =C r^{-(\alpha+\beta)} \int_{M} \int_{B(x, r)}(u(x)-u(y))^{2} d \mu(y) d \mu(x) .
\end{aligned}
$$

Combining this with (6.7), we obtain $\left(R_{1}\right)$, which also implies $\left(R_{\leq}\right)$.

As in the previous part of the proof, $(U E)$ and $\left(R_{1}\right)$ imply that the heat kernel is continuous. Using $(N L E)$ and $\alpha<\beta$ we have

$$
\int_{0}^{\infty} p_{t}(x, y) d t \geq \int_{(d(x, y) / \delta)^{\beta}}^{\infty} c t^{-\alpha / \beta} d t=\infty,
$$

which implies that $(\mathcal{E}, \mathcal{F})$ is recurrent (cf. [11, formula (1.6.2), p. 55]), that is (cf. [11, Theorem 1.6.3, p.58]), there exists a sequence $\left\{u_{n}\right\}_{n=1}^{\infty} \subset \mathcal{F}$ such that

$$
\lim _{n \rightarrow \infty} u_{n}=1 \mu \text {-a.e. and } \lim _{n \rightarrow \infty} \mathcal{E}\left(u_{n}\right)=0 \text {. }
$$

Let $K$ be a compact subset of $M$ and let $x_{0} \in K$. Then there exists an integer $N_{x_{0}}$ such that $u_{n}\left(x_{0}\right) \geq \frac{3}{4}$ for all $n>N_{x_{0}}$. It follows from $\left(R_{1}\right)$ that there exists $\eta$ small 
enough such that $u_{n}(x) \geq \frac{1}{2}$ for all $x \in B\left(x_{0}, \eta\right)$ and all $n>N_{x_{0}}$. Covering $K$ by a finite number of the balls $\left\{B\left(x_{i}, \eta\right)\right\}$, we obtain that $u_{n}(x) \geq \frac{1}{2}$ for all $x \in K$ and all $n \geq N$, for some large integer $N$. Set

$$
v_{n}:=\left(2 u_{n}\right) \wedge 1 \text {. }
$$

We see that $v_{n} \in \mathcal{F}, v_{n}=1$ in $K$ for all $n \geq N$, and

$$
\lim _{n \rightarrow \infty} \mathcal{E}\left(v_{n}\right) \leq 4 \lim _{n \rightarrow \infty} \mathcal{E}\left(u_{n}\right)=0,
$$

whence it follows that

$$
\operatorname{cap}(K) \leq \lim _{n \rightarrow \infty} \mathcal{E}\left(v_{n}\right)=0,
$$

and hence $(\mathcal{E}, \mathcal{F})$ is parabolic.

Finally, let us now prove $\left(R_{\geq}\right)$. Indeed, fix $x, y \in M$. By (6.10) and (6.44) we have, for $x, y \in M$ and $t>0$,

$$
\begin{aligned}
p_{t}(x, x)-p_{t}(x, y) & \leq R(x, y)^{1 / 2} \mathcal{E}\left(p_{t}(x, \cdot)\right)^{1 / 2} \\
& \leq C R(x, y)^{1 / 2} t^{-1 / 2-\alpha /(2 \beta)}
\end{aligned}
$$

On the other hand, it follows from $(N L E),(U E)$ that

$$
\begin{aligned}
p_{t}(x, x)-p_{t}(x, y) & \geq c_{1} t^{-\alpha / \beta}-C_{1} t^{-\alpha / \beta}\left(1+\frac{d(x, y)}{t^{1 / \beta}}\right)^{-(\alpha+\beta)} \\
& \geq \frac{1}{2} c_{1} t^{-\alpha / \beta}
\end{aligned}
$$

provided $d(x, y)=c t^{1 / \beta}$ with a large enough constant $c$. It follows from the above two estimates that

$$
C R(x, y)^{1 / 2} t^{-1 / 2-\alpha /(2 \beta)} \geq \frac{1}{2} c_{1} t^{-\alpha / \beta}
$$

whence

which proves $\left(R_{\geq}\right)$.

$$
R(x, y) \geq\left(\frac{c_{1}}{2 C}\right)^{2} t^{\frac{\beta-\alpha}{\beta}}=c d(x, y)^{\beta-\alpha},
$$

Corollary 6.18. Let $(\mathcal{E}, \mathcal{F})$ be a regular Dirichlet form in $L^{2}(M, \mu)$. Fix parameters $0<\alpha<\beta$ and set $\gamma=\beta-\alpha$. Then the following equivalence is true:

$$
\text { parabolicity }+(V)+\text { "locality" }+(R) \Leftrightarrow\left(U E_{l o c}\right)+(N L E) \text {. }
$$

Proof. Since $\left(U E_{l o c}\right) \Rightarrow(U E)$, we obtain that by Theorem 6.17

$$
\left(U E_{l o c}\right)+(N L E) \Rightarrow \text { parabolicity }+(V)+(R),
$$

whereas $\left(U E_{l o c}\right) \Rightarrow$ ("locality") by [20, Lemma 3.1]. Since the locality is a stronger assumption than $\left(J_{\leq}\right)$, by Theorems 6.17 and 2.3 we have

$$
\text { parabolicity }+(V)+\text { "locality" }+(R) \Rightarrow(U E)+(N L E) \Rightarrow(D U E)+(S) .
$$

Finally, by (1.3) we obtain $\left(U E_{l o c}\right)$.

Remark 6.19. If $(\mathcal{E}, \mathcal{F})$ is parabolic, then $R(x, y)$ is symmetric in $x, y$. Assume in addition that $R$ is a metric in $M$ (called the effective resistance metric). Setting $d(x, y)=R(x, y)$, we see that conditions $\left(R_{1}\right),\left(R_{2}\right)$ and $(R)$ are satisfied with $\gamma=1$. Therefore, Theorem 6.17 implies that

$$
(V)+\left(J_{\leq}\right) \Leftrightarrow(U E)+(N L E),
$$


and Corollary 6.18 implies that

$$
(V)+\left(\text { "locality") } \Leftrightarrow\left(U E_{l o c}\right)+(N L E) .\right.
$$

The latter was also proved in [31, Theorem 3.1] by a probabilistic method.

Example 6.20. Let $M$ be a nested fractal in $\mathbb{R}^{n}$ (cf. 34] that is generated by an iterated function system $\left\{f_{i}\right\}_{i=1}^{N}, N \geq 2$ with contraction ratio $0<s<1$ :

$$
\left|f_{i}(x)-f_{i}(y)\right|=s|x-y| \text { for all } x, y \in \mathbb{R}^{n} .
$$

Let $(\mathcal{E}, \mathcal{F})$ be a local regular Dirichlet form introduced in [27] that is self-similar:

$$
\mathcal{E}(u)=r^{-1} \sum_{k=1}^{N} \mathcal{E}\left(u \circ f_{i}\right),
$$

where $0<r<1$. It was shown that the associated effective resistance $R$ is a metric (cf. [27]). Moreover,

$$
R(x, y) \asymp|x-y|^{\frac{\log r}{\log s}} \text { for all } x, y \in M ;
$$

see [25, p. 163]. If the open set condition holds for $\left\{f_{i}\right\}_{i=1}^{N}$, then the Hausdorff dimension of $M$ is

$$
\alpha=\frac{\log N}{\log (1 / s)},
$$

and the $\alpha$-dimensional Hausdorff measure $\mu$ is $\alpha$-regular. In this case, we see that both conditions $(V)$ and $(R)$ are true. Noting that $(\mathcal{E}, \mathcal{F})$ is parabolic because $1 \in \mathcal{F}$, we obtain $\left(U E_{l o c}\right)$ and $(N L E)$ by using Corollary 6.18, Kumagai 30] obtained the same result by a probabilistic method; see also [21.

\section{Appendix A: Parabolic maximum principle}

Let $(M, d, \mu)$ be a metric measure space. Let $(\mathcal{E}, \mathcal{F})$ be a Dirichlet form in $L^{2}(M, \mu)$ and $\mathcal{L}$ be its generator. Let $I$ be an interval in $\mathbb{R}$, and let $\Omega$ be an open subset of $M$. A function $u: I \rightarrow \mathcal{F}$ is said to be a weak subsolution (resp. a weak supersolution) of the heat equation

$$
\frac{\partial u}{\partial t}+\mathcal{L} u=0
$$

in $I \times \Omega$ if the Frechet derivative $\frac{\partial u}{\partial t}$ of $u$ exists in $I$ in the norm of $L^{2}(\Omega)$ and, for any $t \in I$ and any non-negative function $\psi \in \mathcal{F}(\Omega)$,

$$
\left(\frac{\partial u}{\partial t}(t, \cdot), \psi\right)+\mathcal{E}(u(t, \cdot), \psi) \leq 0 \quad(\text { resp. } \geq 0) .
$$

If the inequality in (7.2) is replaced by equality, then $u$ is called a weak solution of the heat equation (7.1) in $I \times \Omega$. It is known that $P_{t} f$ is a weak solution in $(0, \infty) \times \Omega$ for any open $\Omega \subset M$ (cf. [15, Example 4.10]).

Proposition 7.1 (Parabolic maximum principle 15]). Let u be a weak subsolution of the heat equation in $(0, T) \times \Omega$, where $T \in(0,+\infty]$ and $\Omega$ is an open subset of $M$. Assume in addition that $u$ satisfies the following boundary and initial conditions:

- $u_{+}(t, \cdot) \in \mathcal{F}(\Omega)$ for any $t \in(0, T)$;

- $u_{+}(t, \cdot) \stackrel{L^{2}(\Omega)}{\longrightarrow} 0$ as $t \rightarrow 0$.

Then $u(t, x) \leq 0$ for any $t \in(0, T)$ and $\mu$-almost all $x \in \Omega$. 
Remark 7.2. It was shown in [15, Lemma 4.4] that, for a regular Dirichlet form $(\mathcal{E}, \mathcal{F})$, if $u \in \mathcal{F}$ and if $u \leq v$ for some $v \in \mathcal{F}(\Omega)$, then $u_{+} \in \mathcal{F}(\Omega)$.

Lemma 7.3 (Lemma 4.16, p. 122, 15]). Let $U$ be an open subset of $M$, and $0 \leq f \in L^{2}(U)$. If $u: \mathbb{R}_{+} \rightarrow \mathcal{F}$ is a weak non-negative supersolution to the heat equation in $\mathbb{R}_{+} \times U$ and

$$
u(t, \cdot) \stackrel{L^{2}(U)}{\longrightarrow} f \text { as } t \rightarrow 0
$$

then, for all $t>0$,

$$
u(t, \cdot) \geq P_{t}^{U} f \quad \text { in } M
$$

\section{Appendix B: List of lettered conditions}

Here we list all the lettered conditions used in the text.

Replacing the sign $\asymp$ in condition, say, $(\mathbf{X})$ by $\leq$ and $\geq$, one obtains conditions $\left(\mathbf{X}_{\leq}\right)$and $\left(\mathbf{X}_{\geq}\right)$, respectively. The letters $\alpha, \beta$ always denote fixed positive reals. The constant $\gamma$ is always equal to $\beta-\alpha$ and appears only in the case when $\beta>\alpha$.

$(\mathbf{V})$ : There exist constants $c, C, \alpha>0$ such that, for all $x \in M$ and all $r>0$,

$$
V(x, r) \asymp C r^{\alpha} \text {. }
$$

$(\mathbf{J})$ : The jump density exists and admits the estimate

$$
J(x, y) \asymp C d(x, y)^{-(\alpha+\beta)},
$$

for $\mu$-almost all $x, y \in M$.

(DUE) : On-diagonal upper estimate: the heat kernel $p_{t}$ exists and satisfies the on-diagonal upper estimate

$$
p_{t}(x, y) \leq \frac{C}{t^{\alpha / \beta}},
$$

for all $t>0$ and $\mu$-almost all $x, y \in M$.

(UE) : Upper estimate of non-local type: the heat kernel $p_{t}$ exists and satisfies the off-diagonal upper estimate

$$
p_{t}(x, y) \leq \frac{C}{t^{\alpha / \beta}}\left(1+\frac{d(x, y)}{t^{1 / \beta}}\right)^{-(\alpha+\beta)}
$$

for all $t>0$ and $\mu$-almost all $x, y \in M$.

$\left(\mathbf{U E}_{l o c}\right)$ : Upper estimate of a local type: the heat kernel $p_{t}$ exists and satisfies the off-diagonal upper estimate

$$
p_{t}(x, y) \leq \frac{C}{t^{\alpha / \beta}} \exp \left(-\left(\frac{d(x, y)}{c t^{1 / \beta}}\right)^{\beta /(\beta-1)}\right)
$$

for all $t>0$ and $\mu$-almost all $x, y \in M$ (here $\beta>1$ ).

(UE $\Phi)$ : The heat kernel $p_{t}$ exists and satisfies the estimate

$$
p_{t}(x, y) \leq \frac{C}{t^{\alpha / \beta}} \Phi\left(\frac{d(x, y)}{t^{1 / \beta}}\right)
$$

for all $t>0$ and $\mu$-almost all $x, y \in M$, where $\Phi:[0, \infty) \rightarrow[0, \infty)$ is a continuous, non-increasing function such that

$$
\int_{0}^{\infty} s^{\alpha-1} \Phi(s) d s<\infty .
$$


(NLE) : (The near diagonal lower estimate) There exists $\delta \in(0,1)$ such that, for all $t>0$ and almost all $x, y \in M$ such that $d(x, y) \leq \delta t^{1 / \beta}$,

$$
p_{t}(x, y) \geq c t^{-\alpha / \beta} \text {. }
$$

(S) : Survival estimate. There exist constants $\varepsilon, \delta \in(0,1)$ such that, for all balls $B=B\left(x_{0}, r\right)$ and for all $t^{1 / \beta} \leq \delta r$,

$$
1-P_{t}^{B} \mathbf{1}_{B}(x) \leq \varepsilon \text { for } \mu \text {-almost all } x \in \frac{1}{4} B .
$$

(T) : Tail estimate. There exist constants $\varepsilon, \delta \in(0,1)$ such that, for all balls $B=B\left(x_{0}, r\right)$ and for all $t^{1 / \beta} \leq \delta r$,

$$
P_{t} \mathbf{1}_{B^{c}}(x) \leq \varepsilon \text { for } \mu \text {-almost all } x \in \frac{1}{4} B .
$$

$\left(\mathbf{T}_{\text {strong }}\right)$ : Strong tail estimate. There exist constants $c>0$ and $\beta>0$ such that, for all balls $B=B\left(x_{0}, r\right)$ and for all $t>0$,

$$
P_{t} \mathbf{1}_{B^{c}}(x) \leq \frac{c t}{r^{\beta}} \text { for } \mu \text {-almost all } x \in \frac{1}{4} B .
$$

$\left(\mathbf{R}_{1}\right)$ : For all $u \in \mathcal{F} \cap C_{0}$ and all $x, y \in M$, the following inequality holds:

$$
|u(x)-u(y)|^{2} \leq C d(x, y)^{\gamma} \mathcal{E}(u) .
$$

$\left(\mathbf{R}_{2}\right):$ For all $x \in M$ and $r>0$,

$$
R\left(x, B(x, r)^{c}\right) \geq C^{-1} r^{\gamma} .
$$

(R) : For all $x, y \in M$,

$$
R(x, y) \asymp C d(x, y)^{\gamma} .
$$

\section{REFERENCES}

[1] D. G. Aronson, Non-negative solutions of linear parabolic equations, Ann. Scuola Norm. Sup. Pisa (3) 22 (1968), 607-694. MR0435594 (55 \#8553)

[2] Martin T. Barlow, Diffusions on fractals, Lectures on probability theory and statistics (SaintFlour, 1995), Lecture Notes in Math., vol. 1690, Springer, Berlin, 1998, pp. 1-121, DOI 10.1007/BFb0092537. MR.1668115 (2000a:60148)

[3] Martin T. Barlow and Richard F. Bass, Brownian motion and harmonic analysis on Sierpinski carpets, Canad. J. Math. 51 (1999), no. 4, 673-744, DOI 10.4153/CJM-1999-031-4. MR.1701339(2000i:60083)

[4] Martin T. Barlow, Richard F. Bass, Zhen-Qing Chen, and Moritz Kassmann, Non-local Dirichlet forms and symmetric jump processes, Trans. Amer. Math. Soc. 361 (2009), no. 4, 1963-1999, DOI 10.1090/S0002-9947-08-04544-3. MR2465826 (2010e:60163)

[5] Martin T. Barlow, Thierry Coulhon, and Takashi Kumagai, Characterization of sub-Gaussian heat kernel estimates on strongly recurrent graphs, Comm. Pure Appl. Math. 58 (2005), no. 12, 1642-1677, DOI 10.1002/cpa.20091. MR2177164(2006i:60106)

[6] Martin T. Barlow, Alexander Grigor'yan, and Takashi Kumagai, Heat kernel upper bounds for jump processes and the first exit time, J. Reine Angew. Math. 626 (2009), 135-157, DOI 10.1515/CRELLE.2009.005. MR2492992 (2009m:58077)

[7] Richard F. Bass and David A. Levin, Transition probabilities for symmetric jump processes, Trans. Amer. Math. Soc. 354 (2002), no. 7, 2933-2953 (electronic), DOI 10.1090/S0002-994702-02998-7. MR 1895210 (2002m:60132)

[8] E. A. Carlen, S. Kusuoka, and D. W. Stroock, Upper bounds for symmetric Markov transition functions (English, with French summary), Ann. Inst. H. Poincaré Probab. Statist. 23 (1987), no. 2, suppl., 245-287. MR898496 (88i:35066) 
[9] Zhen-Qing Chen and Takashi Kumagai, Heat kernel estimates for stable-like processes on $d$ sets, Stochastic Process. Appl. 108 (2003), no. 1, 27-62, DOI 10.1016/S0304-4149(03)00105-4. MR2008600 (2005d:60135)

[10] Zhen-Qing Chen and Takashi Kumagai, Heat kernel estimates for jump processes of mixed types on metric measure spaces, Probab. Theory Related Fields 140 (2008), no. 1-2, 277-317, DOI 10.1007/s00440-007-0070-5. MR2357678(2009e:60186)

[11] Masatoshi Fukushima, Yoichi Oshima, and Masayoshi Takeda, Dirichlet forms and symmetric Markov processes, Second revised and extended edition, de Gruyter Studies in Mathematics, vol. 19, Walter de Gruyter \& Co., Berlin, 2011. MR2778606 (2011k:60249)

[12] Alexander Grigor'yan, Analytic and geometric background of recurrence and non-explosion of the Brownian motion on Riemannian manifolds, Bull. Amer. Math. Soc. (N.S.) 36 (1999), no. 2, 135-249, DOI 10.1090/S0273-0979-99-00776-4. MR.1659871 (99k:58195)

[13] Alexander Grigor'yan, Heat kernels and function theory on metric measure spaces (Paris, 2002), Contemp. Math., vol. 338, Amer. Math. Soc., Providence, RI, 2003, pp. 143-172, DOI 10.1090/conm/338/06073. MR2039954(2005c:60096)

[14] Alexander Grigor'yan, Heat kernels on weighted manifolds and applications, The ubiquitous heat kernel, Contemp. Math., vol. 398, Amer. Math. Soc., Providence, RI, 2006, pp. 93-191, DOI 10.1090/conm/398/07486. MR2218016(2007a:58028)

[15] Alexander Grigor'yan and Jiaxin Hu, Off-diagonal upper estimates for the heat kernel of the Dirichlet forms on metric spaces, Invent. Math. 174 (2008), no. 1, 81-126, DOI 10.1007/s00222-008-0135-9. MR2430977 (2009g:58029)

[16] A. Grigor'yan and J. Hu, Upper bounds of heat kernels on doubling spaces, Moscow Math. J. 14 (2014), no. 3, 505-563.

[17] Alexander Grigor'yan, Jiaxin Hu, and Ka-Sing Lau, Heat kernels on metric measure spaces and an application to semilinear elliptic equations, Trans. Amer. Math. Soc. 355 (2003), no. 5, 2065-2095 (electronic), DOI 10.1090/S0002-9947-03-03211-2. MR1953538 (2003j:60103)

[18] Alexander Grigor'yan, Jiaxin Hu, and Ka-Sing Lau, Comparison inequalities for heat semigroups and heat kernels on metric measure spaces, J. Funct. Anal. 259 (2010), no. 10, 26132641, DOI 10.1016/j.jfa.2010.07.010. MR2679020(2012c:58059)

[19] Alexander Grigor'yan, Xueping Huang, and Jun Masamune, On stochastic completeness of jump processes, Math. Z. 271 (2012), no. 3-4, 1211-1239, DOI 10.1007/s00209-011-0911-x. MR2945605

[20] Alexander Grigor'yan and Takashi Kumagai, On the dichotomy in the heat kernel two sided estimates, Analysis on graphs and its applications, Proc. Sympos. Pure Math., vol. 77, Amer. Math. Soc., Providence, RI, 2008, pp. 199-210. MR2459870(2010j:31012)

[21] B. M. Hambly and T. Kumagai, Transition density estimates for diffusion processes on post critically finite self-similar fractals, Proc. London Math. Soc. (3) 78 (1999), no. 2, 431-458, DOI 10.1112/S0024611599001744. MR1665249 (99m:60118)

[22] Ben M. Hambly and Takashi Kumagai, Heat kernel estimates for symmetric random walks on a class of fractal graphs and stability under rough isometries, Fractal geometry and applications: a jubilee of Benoît Mandelbrot, Part 2, Proc. Sympos. Pure Math., vol. 72, Amer. Math. Soc., Providence, RI, 2004, pp. 233-259. MR2112125 (2005k:60141)

[23] Jiaxin $\mathrm{Hu}$, An analytical approach to heat kernel estimates on strongly recurrent metric spaces, Proc. Edinb. Math. Soc. (2) 51 (2008), no. 1, 171-199, DOI 10.1017/S001309150500177X. MR2391637(2009a:58026)

[24] Jiaxin $\mathrm{Hu}$ and Takashi Kumagai, Nash-type inequalities and heat kernels for non-local Dirichlet forms, Kyushu J. Math. 60 (2006), no. 2, 245-265, DOI 10.2206/kyushujm.60.245. MR2268236 (2008d:60102)

[25] Jiaxin $\mathrm{Hu}$ and Xingsheng Wang, Domains of Dirichlet forms and effective resistance estimates on p.c.f. fractals, Studia Math. 177 (2006), no. 2, 153-172, DOI 10.4064/sm177-2-5. MR2285238 (2009j:28021)

[26] J. Hu and M. Zähle, Generalized Bessel and Riesz potentials on metric measure spaces, Potential Anal. 30 (2009), no. 4, 315-340, DOI 10.1007/s11118-009-9117-9. MR2491456 (2010j:31014)

[27] Jun Kigami, Analysis on fractals, Cambridge Tracts in Mathematics, vol. 143, Cambridge University Press, Cambridge, 2001. MR.1840042 (2002c:28015)

[28] Jun Kigami, Harmonic analysis for resistance forms, J. Funct. Anal. 204 (2003), no. 2, 399-444, DOI 10.1016/S0022-1236(02)00149-0. MR2017320(2004m:31010) 
[29] Jun Kigami, Volume doubling measures and heat kernel estimates on self-similar sets, Mem. Amer. Math. Soc. 199 (2009), no. 932, viii+94. MR2512802(2010e:28007)

[30] Takashi Kumagai, Estimates of transition densities for Brownian motion on nested fractals, Probab. Theory Related Fields 96 (1993), no. 2, 205-224, DOI 10.1007/BF01192133. MR $1227032(94 \mathrm{e}: 60068)$

[31] Takashi Kumagai, Heat kernel estimates and parabolic Harnack inequalities on graphs and resistance forms, Publ. Res. Inst. Math. Sci. 40 (2004), no. 3, 793-818. MR2074701 (2005g:60122)

[32] Shigeo Kusuoka, A diffusion process on a fractal, Probabilistic methods in mathematical physics (Katata/Kyoto, 1985), Academic Press, Boston, MA, 1987, pp. 251-274. MR.933827 (89e:60149)

[33] Peter Li and Shing-Tung Yau, On the parabolic kernel of the Schrödinger operator, Acta Math. 156 (1986), no. 3-4, 153-201, DOI 10.1007/BF02399203. MR834612 (87f:58156)

[34] Tom Lindstrøm, Brownian motion on nested fractals, Mem. Amer. Math. Soc. 83 (1990), no. 420 , iv+128. MR988082 (90k:60157)

[35] Jun Masamune and Toshihiro Uemura, Conservation property of symmetric jump processes (English, with English and French summaries), Ann. Inst. Henri Poincaré Probab. Stat. 47 (2011), no. 3, 650-662, DOI 10.1214/09-AIHP368. MR.2841069 (2012f:60291)

[36] Andrzej Stós, Symmetric $\alpha$-stable processes on d-sets, Bull. Polish Acad. Sci. Math. 48 (2000), no. 3, 237-245. MR.1779007 (2002f:60152)

Fakultät für Mathematik, Universität Bielefeld, Postfach 100131, 33501 Bielefeld, Germany

E-mail address: grigor@math.uni-bielefeld.de

Department of Mathematical Sciences, and Mathematical Sciences Center, Tsinghua University, Beijing 100084, People's Republic of China

E-mail address: hujiaxin@mail.tsinghua.edu.cn

Department of Mathematics, The Chinese University of Hong Kong, Shatin, N.T., HONG KONG

E-mail address: kslau@math.cuhk.edu.hk 